\title{
CFT and topological recursion
}

\author{
Ivan Kostov, ${ }^{a, 1}$ and Nicolas Orantin ${ }^{b}$ \\ ${ }^{a}$ Institut de Physique Théorique, CNRS-URA 2306, \\ C.E.A.-Saclay, F-91191 Gif-sur-Yvette, France \\ ${ }^{b}$ Theory division, CERN, \\ CH-1211 Geneva 23, Switzerland \\ E-mail: Ivan.Kostov@cea.fr, Nicolas.Orantin@cern.ch
}

ABSTRACT: We study the quasiclassical expansion associated with a complex curve. In a more specific context this is the $1 / N$ expansion in $\mathrm{U}(N)$-invariant matrix integrals. We compare two approaches, the CFT approach and the topological recursion, and show their equivalence. The CFT approach reformulates the problem in terms of a conformal field theory on a Riemann surface, while the topological recursion is based on a recurrence equation for the observables representing symplectic invariants on the complex curve. The two approaches lead to two different graph expansions, one of which can be obtained as a partial resummation of the other.

KeYwords: Matrix Models, 1/N Expansion

ARXiv EPRINT: 1006.2028

\footnotetext{
${ }^{1}$ Associate member of the Institute for Nuclear Research and Nuclear Energy, Bulgarian Academy of Sciences, 72 Tsarigradsko Chaussée, 1784 Sofia, Bulgaria.
} 


\section{Contents}

1 Introduction $\quad 1$

2 Spectral curve and classical free energy 3

3 The CFT approach $\quad 5$

3.1 Gaussian field on the spectral curve 6

3.2 Operator formalism for the gaussian field on a Riemann surface 6

$\begin{array}{lll}3.3 & \text { Conformal invariance at the branch points } & 8\end{array}$

3.4 Dressing operators 9

3.5 Summary: a universal formula for the $\hbar$ expansion 11

$\begin{array}{lll}3.6 & \text { Diagram technique } & 13\end{array}$

4 Topological recursion $\quad 14$

$\begin{array}{lll}4.1 & \text { Summary } & 14\end{array}$

4.2 Topological recursion in the vicinity of a branch point 15

4.2.1 Solution of the recursion equation for the Kontsevich model 15

$\begin{array}{lll}4.2 .2 & \text { Open-close duality } & 16\end{array}$

4.2.3 The topological recursion for the open coefficients: a trivalent theory 17

$\begin{array}{ll}\text { 4.2.4 Topological recursion for generic values of the moduli } & 19\end{array}$

4.3 Topological recursion for an arbitrary spectral curve 20

$\begin{array}{lll}\text { 4.3.1 Loop insertion operator } & 22\end{array}$

4.3.2 Topological recursion for generic background 22

5 Relation between the two approaches $\quad 22$

6 Conclusion $\quad 24$

$\begin{array}{ll}\text { A Solving the Virasoro constraints near a branch point } & 25\end{array}$

\section{Introduction}

The $1 / N$, or topological, expansion in $\mathrm{U}(N)$ invariant matrix models is being considered with renewed interest in the last years because of its various applications in topological string theories on special classes of Calabi-Yau geometries or $\mathcal{N}=2$ superconformal gauge theories [1-3]. The solution in the limit $N \rightarrow \infty$ is generically described by a complex curve. This is so also in the case when the symmetry group is a direct product of several unitary groups, as the ADE matrix chains $[4,5]$. Since the complex curve determines the classical spectral density of the matrix variables, it is also called spectral curve. In all solved examples, the $1 / N$ expansion can be formulated entirely in terms of the complex curve. 
The fact that the spectral curve determines not only the classical limit $N \rightarrow \infty$, but also the complete $1 / N$ expansion, is highly non-trivial and deserves to be better understood.

Two approaches to the $1 / N$ expansion formulated in terms of the spectral curve were developed in the past ten years. The first approach, which will be refered to as CFT approach, is based on the conformal invariance, which is believed to be present in all $\mathrm{U}(N)$ invariant matrix systems. The conformal symmetry is obvious for the class of matrix models which, after diagonalization, reduce to Coulomb gases [6-8]. A prescription to evaluate the quasiclassical expansion in such matrix models using the toolbox of CFT was outlined by one of the authors in $[8,9]$. The basic idea is that the conformal invariance is sufficient to construct the collective field to all orders in the quasiclassical expansion. The spectral curve appears as the classical value of a bosonic field, which lives on a Riemann surface associated with this spectral curve. The gaussian approximation gives the two leading terms of the $1 / N$ expansion, while the higher terms are obtained by inserting special local operators at the branch points of the Riemann surface. ${ }^{1}$ The proposal of $[8,9]$ is a field-theoretical formulation of the 'method of moments' for performing the higher genus calculations, developped in [11], and generalizes the CFT description of the non-critical string theories [12-14]. The case of a hyperelliptic Riemann surfaces was considered in details in [15].

Another approach to the $1 / N$ expansion, known as topological recursion, was developed more recently by B. Eynard and collaborators [16-26]. Whilst the CFT approach is related to field theory, the topological recursion, which we denote shortly by TR, is related to algebraic geometry. The topological recursion gives a very efficient algorithm for calculating the $1 / N$ expansion of the observables representing symplectic invariants on the complex curve. The recursion procedure overcomes some of the technical difficulties of the method of moments [11]. The basic observation here is that recurrence equation is most simply formulated in terms of residues at the branch points of the Riemann surface. The outcome of the topological recursion is an elegant graphical scheme, containing only trivalent vertices.

Let us emphasize that the CFT and TR approaches can be formulated only in terms of the spectral curve, without any reference to the underlying matrix model. Therefore, instead of speaking of the $1 / N$ expansion, we will speak of the quasiclassical expansion (in $\hbar=1 / N)$ associated with the spectral curve. Since any spectral curve can be considered as a solution of the universal Whitham hierarchy [27], CFT and TR give two methods to evaluate the quasiclassical expansion of an integrable hierarchy, given its dispersionless limit. $^{2}$

The aim of this paper is to compare the two methods and the diagram techniques they

\footnotetext{
${ }^{1}$ The field-theoretical description of the branch points was pioneered by Al. Zamolodchikov [10].

${ }^{2}$ Reconstruction of the quasiclassical expansion for the KP hierarchy, named $\hbar$-dependent KP hierarchy, was considered in $[28,29]$. A serious problem in these works, which are based on the Lax formalism, is that the recursion relations are extremely complicated. The advantage of the CFT/TR method, based on the Virasoro symmetry, is that the solution is obtained in a simple and easy to manipulate form. We are not going to discuss here the uniqueness of the quasiclassical expansion. CFT/TR is believed to give the same quasiclassical expansion as the Lax formalism, but as far as we know there is no general proof of that.
} 
lead to. Since the CFT method and the topological recursion represent two different techniques to resolve the Virasoro constraints, they are expected to lead to the same solution, although this is far from obvious for general spectral curve. On the other hand, the two diagram techniques are seemingly very different and have different geometrical interpretation in terms of world sheets. Therefore we think that it is important and instructive to find out the exact correspondence between the two forms of the quasiclassical expansion associated with a spectral curve. In this paper we show that the two diagram techniques lead to the same result and that moreover one of them is obtained by a partial resummation of the other. We conclude that the CFT method and the topological recursion are two different realizations of the same procedure, which we will refer to as CFT/TR. We underline that we are considering the case of the most general, not necessarily hyperelliptic, compact complex curve. ${ }^{3}$

The paper is organized as follows. In section 2 we collect some definitions and notations related to the spectral curve. In section 3 we describe the CFT approach. We first remind the operator formalism for a gaussian field on a Riemann surface. Then we express the Virasoro constraints the operator formalism for a gaussian field on the spectral curve. We write the Virasoro constraints in terms of the mode expansion of the gaussian field near the branch points and construct the dressing operators to be inserted at the branch points in terms of these modes. Finally we derive the diagram technique for computing the higher genus free energies and correlation functions. In section 4 we first give a brief summary of the topological recursion. Then we rewrite the equations of TR in terms of the series expansions of the symplectic invariants near the branch points. We start with the simplest case of a spectral curve with only one, simple, branch point. In this case the symplectic invariants are given by the observables of the Kontsevich model. We formulate the equations of TR in terms of a trivalent graph expansion. After that we consider the case of an arbitrary spectral curve. Section 5 we give the exact correspondence between the diagram techniques in the CFT and the TR approaches.

\section{Spectral curve and classical free energy}

By spectral curve we understand the triple

$$
\mathcal{E} \stackrel{\text { def }}{=}(\Sigma, x, y)
$$

where $\Sigma$ is a Riemann surface of genus $G$ and the functions $x$ and $y$ are analytic in some open domain of $\Sigma$. The Riemann surface is assumed to have $l$ punctures $(l \geq 1)$, where the differential $y d x$ becomes singular. For later convenience we introduce a global parametrization $z$ of the Riemann surface $\Sigma$. The complex variable $z$ belongs either to the Riemann sphere (for genus 0) or to a quotient of the unit disk (for genus 1 or larger). In terms of the global parameter $z$ we have

$$
x=x(z), \quad y=y(z),
$$

\footnotetext{
${ }^{3}$ From this perspective, the result of this paper goes beyond the unitary one matrix model. It applies for example ti the unitary two matrix models where the spectral curve is not hyperelliptic in general.
} 
where $y$ and $x$ are analytic functions of $z$. In the neighborhood of the punctures $z=\hat{a}_{\alpha}$ we chose local coordinates $\zeta_{(z, \alpha)}$ such that

$$
\zeta_{\left(\hat{a}_{\alpha}, \alpha\right)}^{-1}=0 \quad(a=1, \ldots, l) .
$$

We can turn the spectral curve into a set of algebro-geometric data for Krichever's universal Whitham hierarchy [27]. We introduce the classical collective field as the one-form

$$
d S(z)=y(z) d x(z)
$$

which is by definition holomorphic on the Riemann surface except at the punctures $z=\hat{a}_{\alpha}$. The function $S(x)$ is nothing but the potential for the universal Whitham hierarchy. The classical free energy associated with the spectral curve is defined as [27]

$$
\mathcal{F}^{(0)}[S]=\int_{\Sigma} \bar{d} S \wedge d S .
$$

The deformations of the spectral curve can be represented as commuting flows in the phase space corresponding to the "times" associated with the punctures. The Poisson brackets defined in the space of functions of the two variables $x$ and $y$ are defined as

$$
\{f, g\} \stackrel{\text { def }}{=} \partial_{x} f \partial_{y} g-\partial_{x} g \partial_{y} f
$$

and correspond to the symplectic form $\omega=d x \wedge d y$. The classical field $S$ can be thought of as the action of a Hamiltonian system depending on the coordinate $x$ and the "times" associated with the expansion at the punctures [27].

The classical problem in the universal Whitham hierarchy is to determine the oneform (2.4) by its asymptotics at the punctures and the moduli associated with the noncontractible cycles. The moduli of the spectral curve at the punctures are given by the principal parts

$$
t_{\alpha} \equiv \operatorname{Res}_{z \rightarrow \hat{a}_{\alpha}} y(z) d x(z)
$$

and the potentials

$$
V_{\alpha}(z) \equiv \operatorname{Res}_{z^{\prime} \rightarrow \hat{a}_{\alpha}} y\left(z^{\prime}\right) d x\left(z^{\prime}\right) \ln \left(1-\frac{\zeta_{(z, \alpha)}}{\zeta_{\left(z^{\prime}, \alpha\right)}}\right) .
$$

Let $\left\{\mathcal{A}_{i}, \mathcal{B}_{i}\right\}_{i=1}^{G}$ be a canonical basis of cycles on $\Sigma$. The classical solution is completely determined by its asymptotics at the punctures and the 'filling fractions'

$$
\nu_{k}=\frac{1}{2 \pi i} \oint_{\mathcal{A}_{k}} y(z) d x(z) \quad(k=1, \ldots, G)
$$

associated with the A-cycles. A basis of holomorphic one-forms on the Riemann surface associated with the cycles $\mathcal{A}_{k}$ is given by the derivatives

$$
\omega_{k}=\frac{\partial}{\partial \nu_{k}} y d x
$$


Their integrals along the A- and B-cycles are given by

$$
\frac{1}{2 \pi i} \oint_{\mathcal{A}_{k}} \omega_{l}=\delta_{k l}, \quad \frac{1}{2 \pi i} \oint_{\mathcal{B}_{k}} \omega_{l}=\tau_{k l}
$$

where $\tau=\left\{\tau_{i k}\right\}$ is the period matrix of the Riemann surface. We will consider the filling fractions as a set of external parameters, which are part of the moduli of the spectral curve.

An important role in the construction of the quasiclassical expansion is played by the Bergman kernel for the spectral curve, $B\left(z, z^{\prime}\right)$. The Bergman kernel is defined uniquely by the following three conditions:

- it is globally defined on the Riemann surface, including the punctures;

- it behaves at small distances as

$$
B\left(z, z^{\prime}\right)=\frac{d z d z^{\prime}}{\left(z-z^{\prime}\right)^{2}}+\text { regular function; }
$$

- it has has vanishing integrals around the $\mathcal{A}$-cycles:

$$
\oint_{\mathcal{A}_{i}} B\left(z, z^{\prime}\right)=0, \quad(j=1, \ldots, G) .
$$

The Bergman kernel (2.12) depends on the spectral curve only through the $3 G-3+l$ (for $G \geq 2$ ) complex moduli of the punctured Riemann surface $\Sigma$. It is important noticing that it does not depend on the moduli associated with the singular behavior of $y d x$ near the punctures.

Below we will assume that the classical problem is solved and the one-form (2.4) is already known. The free energy (2.5), or the classical action for the collective field, can be written as a sum of residues associated with the punctures and contour integrals of the one-form $y d x[19,27,30]$. For the sake of completeness we remind the general expression for $\mathcal{F}^{(0)}[S]$, although we will not use it:

$$
\mathcal{F}^{(0)}[S]=-\frac{1}{2}\left[\sum_{\alpha} \operatorname{Res}_{z \rightarrow \hat{a}_{\alpha}} V_{\alpha}(z) y(z) d x(z)+\sum_{\alpha} t_{\alpha} \mu_{\alpha}+\sum_{i=1}^{G} \nu_{i} \oint_{\mathcal{B}_{i}} y(z) d x(z)\right],
$$

where

$$
\mu_{\alpha} \equiv \int_{\left(z=\hat{a}_{\alpha}\right)}^{o}\left(y(x) d x(x)-d V_{\alpha}(z)+t_{\alpha} \frac{d \zeta_{(z, \alpha)}}{\zeta_{(z, \alpha)}}\right)+V_{\alpha}(o)-t_{\alpha} \ln \left(\zeta_{(o, \alpha)}\right)
$$

for an arbitrary base point $o$.

\section{The CFT approach}

The CFT approach is based on the assumption that the quantum collective field, which we denote by $\Phi$, has the same analytical properties as the classical solution $S(x)$. In other words, the field $\Phi$ is invariant with respect to the conformal transformations of the 
spectral parameter $x$ that preserve the $l$ punctures $\hat{x}_{\alpha}=x\left(\hat{a}_{\alpha}\right)$ of the Riemann surface. ${ }^{4}$ The outcome of the CFT approach is an universal formula for the quasiclassical expansion of the free energy

$$
\mathcal{F}[S, \hbar]=\sum_{g \geq 0} \hbar^{2 g-2} \mathcal{F}^{(g)}[S]+\text { non-perturbative terms } .
$$

in terms of the classical solution $S(x)$.

\subsection{Gaussian field on the spectral curve}

In the gaussian approximation, the classical field $\Phi_{\mathrm{cl}}$ is the expectation value of a gaussian field $\Phi$. We thus split the collective field into classical and quantum parts:

$$
\Phi=\Phi_{\mathrm{cl}}+\Phi_{\mathrm{qu}}, \quad \Phi_{\mathrm{cl}}=\frac{1}{\hbar} S
$$

The subleading term in the quasiclassical expansion for the free energy, $\mathcal{F}_{\text {gauss }}=\mathcal{F}^{(1)}$, comes from the gaussian fluctuations around the classical solution. The corresponding factor in the partition function is given by the inverse power of the holomorphic piece of the determinant of the Laplace operator on the Riemann surface:

$$
\mathcal{Z}_{\text {gauss }}=\frac{1}{\operatorname{det} \bar{\partial}_{0}}
$$

The expression of the chiral determinant for a hyperelliptic curve has been given in [10] and for a general surface in [31, 32].

All correlation functions in the gaussian approximation can be expressed through the two-point function of a gaussian field on the Riemann surface, which is given by the Bergmann kernel,

$$
B\left(z, z^{\prime}\right)=\left\langle d \Phi_{\mathrm{qu}}(z) d \Phi_{\mathrm{qu}}\left(z^{\prime}\right)\right\rangle_{\Sigma}
$$

\subsection{Operator formalism for the gaussian field on a Riemann surface}

Let $z_{0}$ be a base point on the Riemann surface $\Sigma$ and $\xi_{(z)}$ be a local coordinate in the neighborhood of $z_{0}$ such that $\xi_{\left(z_{0}\right)}=0$. We can associate with this variable a Hilbert space by expanding the gaussian field in Laurent series. We split the quantum field into a singular and a regular parts

$$
d \Phi_{\mathrm{qu}}(z)=d \Phi_{\mathrm{qu}}(z)^{\mathrm{sing}}+d \Phi_{\mathrm{qu}}(z)^{\mathrm{reg}}
$$

having mode expansions

$$
d \Phi_{\mathrm{qu}}(z)^{\mathrm{sing}}=\sum_{n \geq 0} J_{n} \xi_{(z)}^{-n-1} d \xi_{(z)}, \quad d \Phi(z)_{\mathrm{qu}}^{\mathrm{reg}}=\sum_{n \geq 1} J_{n} \xi_{(z)}^{n-1} d \xi_{(z)} .
$$

\footnotetext{
${ }^{4}$ The invariance with respect to conformal transformations which do not preserve the punctures determines the reaction of the system to a change of the external parameters. This symmetry is not relevant for the quasiclassical expansion.
} 
The amplitudes $J_{n}$ are assumed to satisfy canonical commutation relations

$$
\left[J_{m}, J_{n}\right]=m \delta_{m+n, 0}, \quad m, n \in \mathbb{Z} .
$$

This mode expansion defines a Hilbert space with left and right Fock vacua satisfying ${ }^{5}$

$$
\left\langle 0\left|J_{-n}=0, \quad J_{n}\right| 0\right\rangle=0 \quad(n \geq 1) .
$$

From the commutation relation

$$
\left[d \Phi_{\mathrm{qu}}(z)^{\mathrm{sing}}, d \Phi_{\mathrm{qu}}^{\mathrm{reg}}\left(z^{\prime}\right)\right]=\frac{d \xi_{(z)} d \xi_{\left(z^{\prime}\right)}}{\left[\xi_{(z)}-\xi_{\left(z^{\prime}\right)}\right]^{2}},
$$

one finds for the correlation function

$$
\left\langle 0\left|d \Phi_{\mathrm{qu}}(z) d \Phi_{\mathrm{qu}}\left(z^{\prime}\right)\right| 0\right\rangle=\frac{d \xi_{(z)} d \xi_{\left(z^{\prime}\right)}}{\left[\xi_{(z)}-\xi_{\left(z^{\prime}\right)}\right]^{2}}
$$

The left and right Fock vacua defined above are such that the gaussian field $\Phi_{\mathrm{qu}}$ has vanishing expectation value and its two-point function (3.10) gives the singular part of the Bergman kernel. We would like to deform the left and right vacuum states in such a way that the two-point function of $\Phi_{\text {qu }}$ reproduces also the regular part of the Bergman kernel (2.12). In order to obtain the operator representation of the collective field $\Phi$, eq. (3.2), we should also introduce the expectation expectation value $\Phi_{\mathrm{cl}}$. For that we first expand the classical field and the Bergman kernel in the local variable $\xi_{(z)}$, assuming that $\Phi_{\mathrm{cl}}^{\text {sing }}\left(z_{0}\right)=0$, i.e. $z_{0}$ is a regular point on the Riemann surface:

$$
\begin{aligned}
& d \Phi_{\mathrm{cl}}(z)=\frac{1}{\hbar} \sum_{n \geq 1} \mu_{n} \xi_{(z)}^{n-1} d \xi_{(z)}, \\
& B\left(z, z^{\prime}\right)=\frac{d \xi_{(z)} d \xi_{\left(z^{\prime}\right)}}{\left[\xi_{(z)}-\xi_{\left(z^{\prime}\right)}\right]^{2}}+\sum_{n, m \geq 1} B_{m, n} \xi_{(z)}^{n-1} \xi_{\left(z^{\prime}\right)}^{m-1} d \xi_{(z)} d \xi_{\left(z^{\prime}\right)} .
\end{aligned}
$$

Then, as suggested in [33-35], we Bogolyubov transform the left Fock vacuum,

$$
\langle 0| \rightarrow\langle\Sigma|=\langle 0| \exp \left(\frac{1}{2} \sum_{m, n \geq 1} \frac{B_{m, n}}{m n} J_{m} J_{n}+\frac{1}{\hbar} \sum_{n \geq 1} \frac{\mu_{n}}{n} J_{n}\right) .
$$

In this way the Riemann surface $\Sigma$ is represented by a state $\langle\Sigma|$ belonging to the Fock space associated with the base point $z_{0}$. For any correlation function of $d \Phi_{\mathrm{qu}}\left(z_{i}\right)$, with $z_{i}$ belonging to a coordinate patch which contains the point $z_{0}$, the unnormalized expectation value on the surface $\Sigma$ is given by

$$
\left\langle d \Phi_{\mathrm{qu}}\left(z_{1}\right) \ldots d \Phi_{\mathrm{qu}}\left(z_{n}\right)\right\rangle_{\Sigma}=\left\langle\Sigma\left|d \Phi_{\mathrm{qu}}\left(z_{1}\right) \ldots d \Phi_{\mathrm{qu}}\left(z_{n}\right)\right| 0\right\rangle .
$$

\footnotetext{
${ }^{5}$ The amplitude $J_{0}$ commutes with the rest and its action on the vacuum states can be specified separately. We can define a vacuum state with charge $N$ by requiring that $J_{0}|0\rangle=N|0\rangle$. Alternatively one can introduce the zero mode $\Phi_{0}$, which is canonically conjugated to $J_{0}$, so that $\left[J_{0}, \Phi_{0}\right]=1$. Then the vacuum state with charge $N$ is $e^{N \Phi_{0}}|0\rangle$.
} 
If we need to evaluate the correlation functions of operators that belong to different coordinate patches of $\Sigma$, we should generalize the operator representation for a Riemann surface with several extra punctures $z_{1}, \ldots, z_{r}$. To each puncture we associate a pair of vacuum states as above and define the right and the left Fock vacua as the direct products of the Fock vacua associated with each puncture. Let $\xi_{(z, s)}$ be the local coordinate in the $s$-th coordinate patch. Then (3.13) generalizes to

$$
\langle 0|=\bigotimes_{s=1}^{r}\left\langle 0^{(s)}\right| \rightarrow\langle\Sigma|=\langle 0| \exp \left(\frac{1}{2} \sum_{m, n \geq 1} \frac{1}{m n} B_{m, n}^{\left(s, s^{\prime}\right)} J_{m}^{(s)} J_{n}^{\left(s^{\prime}\right)}+\frac{1}{\hbar} \sum_{n \geq 1} \frac{1}{n} \mu_{n}^{(s)} J_{n}^{(s)}\right),
$$

where

$$
\begin{aligned}
\mu_{n}^{(s)} & =\operatorname{Res}_{z \rightarrow z_{s}} y(z) d x(z) \xi_{(z, s)}^{-n}, \\
B_{m, n}^{\left(s, s^{\prime}\right)} & =\operatorname{Res}_{z \rightarrow z_{s}} \operatorname{Res}_{z \rightarrow z_{s^{\prime}}} B\left(z, z^{\prime}\right) \xi_{(z, s)}^{-m} \xi_{\left(z^{\prime}, s^{\prime}\right)}^{-n} .
\end{aligned}
$$

\subsection{Conformal invariance at the branch points}

The stress-energy tensor for the conformal transformations of the global parameter $z$ is

$$
T(z) d z^{2}=\frac{1}{2} \lim _{z^{\prime} \rightarrow z}\left[d \Phi(z) d \Phi\left(z^{\prime}\right)-B\left(z, z^{\prime}\right)\right] .
$$

The gaussian field is by definition invariant under such transformations in the sense that the expectation value $\langle T(z)\rangle_{\Sigma}$ is analytic everywhere except at the punctures $z=\hat{a}_{\alpha}$ where the classical solution has poles.

The basic assumption of the CFT approach is that the theory is invariant with respect to conformal transformations of the spectral variable $x$. The conformal invariance in the $x$-plane leads to a stronger condition,

$$
\left(\frac{d z}{d x}\right)^{2}\langle T(z)\rangle_{\Sigma}=\text { analytic function of } x
$$

with possible singularities at the punctures $\hat{x}_{\alpha}=x\left(\hat{a}_{\alpha}\right)$. The condition (3.17) is satisfied everywhere on the Riemann surface except at the branch points ${ }^{6}$ where $\partial_{z} x=0$. This means that the gaussian field is not a good approximation near the branch points.

If the vicinity of the branch point $a_{s}$ one can define an involution $z \leftrightarrow \tilde{z}$, such that $\tilde{z} \neq z$ and $x(\tilde{z})=x(z)$. Up to quadratic terms in $z-a_{s}, \tilde{z}-a_{s}=a_{s}-z$. In general, the involution $z \leftrightarrow \tilde{z}$ depends on the branch point; it is globally defined only if the spectral curve is hyperelliptic. The Hilbert space associated with base point $z=a_{s}$ splits into odd and even sectors with respect to the involution $z \leftrightarrow \tilde{z}$. If the Riemann surface $\Sigma$ is viewed as a branched cover of the spectral plane $x$, the involution $z \leftrightarrow \tilde{z}$ is the monodromy around the branch point $x_{s}=x\left(a_{s}\right)$. This is why the odd sector will be called twisted sector. ${ }^{7}$

\footnotetext{
${ }^{6}$ Strictly speaking, we should use the term ramification points instead of branch points. According to the standard terminology, the points $a_{s} \in \Sigma$ where $d x(z)=0$ are ramification points while the points $x\left(a_{s}\right) \in \mathbb{C}$ are branch points. Each branch point is the image of one or more ramification points in the $x$-plane.

7 The richer structure of the Hilbert space at the branch points is related to the extended symmetry. For a branch point this is the $\hat{u}(2)=\hat{s} u(2) \oplus \hat{u}(1)$ current algebra. In the case of a branch point of order $m$ the symmetry is $\hat{u}(m)$. The Cartan subalgebra of $\hat{u}(m)$ is spanned by the $m$ currents associated with the different sheets of the Riemann surface near the branch point.
} 
Each sector is characterized by its stress-energy tensor. The stress-energy tensor for the even sector automatically sarisfies (3.17) near the branch point, while the stress-energy tensor for the odd sector does not. We define the twisted component of the collective field as the projection

$$
\Phi^{[s]}(z) \stackrel{\text { def }}{=} \frac{\Phi(z)-\Phi(\tilde{z})}{2} \quad \text { for } z \text { in the vicinity of } a_{s} .
$$

The two-point function and the stress-energy tensor of the twisted field are ${ }^{8}$

$$
\begin{aligned}
\left\langle d \Phi_{\mathrm{qu}}^{[s]}(z) d \Phi_{\mathrm{qu}}^{[s]}\left(z^{\prime}\right)\right\rangle_{\Sigma} & =B^{[s]}\left(z, z^{\prime}\right) \equiv \frac{1}{2} B\left(z, z^{\prime}\right)-\frac{1}{2} B\left(z, \tilde{z}^{\prime}\right), \\
T^{[s]}(z) d z^{2} & =\frac{1}{2} \lim _{z^{\prime} \rightarrow z}\left[d \Phi^{[s]}(z) d \Phi^{[s]}\left(z^{\prime}\right)-B^{[s]}\left(z, z^{\prime}\right)\right] .
\end{aligned}
$$

The condition (3.17) represents a non-trivial constraint for the twisted stress-energy tensor $T^{[s]}(z)$.

We assume that all branch points are simple. Moreover, we assume that these points are neither poles of $y$ neither zeroes of $d y$. If $a_{s}$ is one of the branch points, i.e. $d x / d z \sim$ $\left(z-a_{s}\right),(3.17)$ implies that the Laurent expansion of the stress-energy tensor contains only even powers of $z-a_{s}$,

$$
T^{[s]}(z)=\sum_{n \in \mathbb{Z}} L_{2 n}^{[s]}\left(z-a_{s}\right)^{-2 n-2},
$$

and the non-vanishing Virasoro operators $L_{2 n}$ satisfy

$$
\left\langle L_{2 n}^{[s]}\right\rangle_{\Sigma}=0, \quad n \geq-1 .
$$

\subsection{Dressing operators}

Our aim is to construct dressing operators located at the branch points, which restore the conformal invariance. For that we will apply the operator formulation (3.15)-(3.16) to the special case when the extra punctures are placed at the branch points $z=a_{s}(s=$ $\left.1, \ldots, n_{B}\right)$.

Given a local coordinate $\xi_{(z, s)}$, such that $\xi_{\left(a_{s}, s\right)}=0$, the mode expansion for the twisted field (3.18) is given by the odd part of the mode expansion (3.6),

$$
\begin{aligned}
& d \Phi_{\mathrm{qu}}^{[s]}(z)=\sum_{p \text { odd }} J_{p}^{[s]} \xi_{(z, s)}^{-p-1} d \xi_{(z, s)}, \\
& d \Phi_{\mathrm{cl}}^{[s]}(z)=\frac{1}{\hbar} \sum_{p \geq 3, \text { odd }} \mu_{p}^{[s]} \xi_{(z, s)}^{p-1} d \xi_{(z, s)} .
\end{aligned}
$$

We denote by $\left|0_{\mathrm{tw}}^{[s]}\right\rangle$ the twisted Fock vacuum associated with the branch point $a_{s}$. From the point of view of a CFT on the spectral plane, this state can be considered as the result of the insertion at the point $x_{s}$ of a twist operator of conformal weight $\frac{1}{16}$. The Hilbert

\footnotetext{
${ }^{8}$ Since the function is symmetric with respect to exchanging the two arguments, one can antisymmetrize only with respect of the second argument.
} 
space space associated with the ensemble of the branch points of the Riemann surface is spanned by the states of the form

$$
\left\langle 0_{\mathrm{tw}}\left|\prod_{i} J_{p_{i}}^{\left[s_{i}\right]}, \quad \prod_{i} J_{-p_{i}}^{\left[s_{i}\right]}\right| 0_{\mathrm{tw}}\right\rangle \quad\left(p_{i} \geq 1, \text { odd }\right)
$$

where the left and right Fock vacua are defined as

$$
\left\langle 0_{\mathrm{tw}}\left|=\bigotimes_{s=1}^{n_{B}}\left\langle 0_{\mathrm{tw}}^{[s]}|, \quad| 0_{\mathrm{tw}}\right\rangle=\bigotimes_{s=1}^{n_{B}}\right| 0_{\mathrm{tw}}^{[s]}\right\rangle .
$$

Once we have an operator representation of the expectation value in (3.17), the construction of the dressing operators becomes a purely algebraic problem. The result does not depend on the choice of parametrization, but the mode expansion (3.23) does. Let us pick a canonical parametrization in the vicinity of each branch point. We would like to choose the local parametrization variable $\xi_{(z, s)}$ so that the moments $\mu_{p}^{[s]}$ are simply expressed in terms of the moduli $M_{p}^{[s]}$ of the spectral curve, defined $\mathrm{as}^{9}$

$$
M_{p}^{[s]}=\oint_{a_{s}} \frac{y(z) d x(z)}{\left[x(z)-x\left(a_{s}\right)\right]^{p / 2}}, \quad p=3,5, \ldots
$$

The most convenient choice of $\xi_{(z, s)}$, which we adopt in the following, is

$$
\xi_{(z, s)}=\sqrt{2}\left[M_{p}^{[3]}\right]^{1 / 3} \sqrt{x(z)-x\left(a_{s}\right)} .
$$

Then the moments (3.16) at the branch point $a_{s}$ are given by

$$
\mu_{p}^{[s]}=\left(M_{3}^{[s]}\right)^{-p / 3} M_{p}^{[s]} .
$$

The left state $\langle\Sigma|$ in the operator representation (3.14) is

$$
\langle\Sigma|=\left\langle 0_{\mathrm{tw}}\right| \exp \left(\frac{1}{2} \sum \frac{1}{p q} B_{p, q}^{\left[s, s^{\prime}\right]} J_{p}^{[s]} J_{q}^{\left[s^{\prime}\right]}+\frac{1}{\hbar} \sum \frac{1}{p} \mu_{p}^{[s]} J_{p}^{[s]}\right)
$$

where $s, s^{\prime}$ take values $1, \ldots, n_{B}$ and $p, q$ are positive odd integers. By convention $\mu_{p}^{[s]}=0$ if $p \leq 1$.

We are looking for a right state of the form

$$
|\Omega\rangle=\Omega\left|0_{\mathrm{tw}}\right\rangle, \quad \Omega=\bigotimes_{s=1}^{n_{B}} \Omega^{[s]},
$$

where $\Omega^{[s]}$ is the dressing operator associated with the $s$-th branch point. The operator $\Omega^{[s]}$ is determined from the conformal Ward identity

$$
\left\langle\Sigma\left|L_{2 n}^{[s]} \Omega^{[s]}\right| 0_{\mathrm{tw}}^{[s]}\right\rangle=0 \quad\left(n \geq-1 ; s=1, \ldots, n_{B}\right),
$$

\footnotetext{
${ }^{9}$ These moduli are linear combinations of the ACKM moments [11].
} 
where $L_{2 n}^{[s]}$ are Virasoro operators associated with the expansion of the twisted stress-energy tensor (3.20):

$$
T_{\mathrm{tw}}^{[s]}(z)=\sum_{n \in \mathbb{Z}} L_{2 n}^{[s]} \xi_{(z, s)}^{-2 n-4}
$$

The explicit expression for the Virasoro operators is

$$
L_{2 n}^{[s]}=\frac{1}{4} \sum_{p+q=2 n}: J_{p}^{[s]} J_{q}^{[s]}:+\frac{1}{16} \delta_{n, 0} \quad(n \geq-1) .
$$

In order to satisfy (3.31) it is sufficient to solve the operator equation

$$
e^{\frac{1}{3 \hbar} J_{3}^{[s]}} L_{2 n}^{[s]} \hat{\Omega}^{[s]}\left|0_{\mathrm{tw}}^{[s]}\right\rangle=0 \quad\left(n \geq-1, s=1, \ldots, n_{B}\right) .
$$

Here we retained only the factor $\exp \left(\frac{1}{3 \hbar} \mu_{3}^{[s]} J^{[s]}\right)$, with $\mu_{3}^{[s]}=1$, from the operator deforming the left vacuum in (3.29). This operator, when commuted to the right, shifts $J_{p}^{[s]} \rightarrow$ $J_{p}^{[s]}+\delta_{p+3,0}$, which corresponds to the 'minimal' classical solution having a branch point at $x_{s}: \Phi_{\mathrm{cl}}^{[s]} \sim \hbar^{-1}\left(x-x_{s}\right)^{3 / 2}$.

Assuming that the dressing operator $\Omega^{[s]}$ can be expanded as a formal series in the creation operators $J_{-p}^{[s]}(p \geq 1)$, the solution of (3.34) is given by

$$
\Omega^{[s]}=C^{[s]} \exp \left(\sum_{n \geq 1} \frac{(-1)^{n}}{n !} \sum_{p_{1}, \ldots, p_{n}} \hbar^{2 g-2+n} \frac{w_{p_{1}, \ldots, p_{n}}^{(g)}}{p_{1} \ldots p_{n}} J_{-p_{1}}^{[s]} \ldots J_{-p_{n}}^{[s]}\right)
$$

where $C^{[s]}$ is a numerical factor depending on the moduli at the point $a_{s}$. The sum goes over odd positive odd integers $p_{i}$. The coefficients $w_{p_{1}, \ldots, p_{n}}^{(g)}$ are universal rational numbers and are proportional to the correlation functions in the Kontsevich model [36]. They are nonzero only if the genus $g$ defined by

$$
\frac{1}{3} \sum_{i=1}^{n} p_{i}=2 g-2+n
$$

is a positive integer. For the sake of self-consistency, we derive in appendix A the recurrence equation for $w_{p_{1}, \ldots, p_{n}}^{(g)}$.

The numerical factor $C^{[s]}$ is fixed by the scale invariance. Upon a rescaling $x \rightarrow \rho x$, the twisted vacuum acquires a factor $\rho^{1 / 16}$ and $M_{3}^{[s]} \rightarrow \rho^{-3 / 2} M_{3}^{[s]}$, while the dimensionless moments $\mu_{p}^{[s]}$ do not change. The dressed twisted vacuum remains scale invariant if

$$
C^{[s]}=\left(M_{3}^{[s]}\right)^{-1 / 24}
$$

\subsection{Summary: a universal formula for the $\hbar$ expansion}

\section{Partition function}

The free energy $\mathcal{F}_{\hbar}=\ln \mathcal{Z}_{\hbar}$ is a sum of three terms,

$$
\mathcal{F}_{\hbar}=\mathcal{F}_{\text {cl }}+\mathcal{F}_{\text {gauss }}+\mathcal{F}_{\text {int }}
$$


where $\mathcal{F}_{\mathrm{cl}}=\hbar^{-2} \mathcal{F}^{(0)}$ is the classical action of the gaussian field on the Riemann surface,

$$
\mathcal{F}_{\text {gauss }}=\mathcal{F}^{(1)}=-\ln \operatorname{det} \bar{\partial}-\frac{1}{24} \sum_{s=1}^{n_{B}} \ln M_{3}^{[s]}
$$

is the sum of the gaussian fluctuations and the contributions from the scaling factors associated with the branch points, and $\mathcal{F}_{\text {int }}$ is the interaction part, which vanishes when $\hbar \rightarrow 0$.

Assuming that $\mathcal{F}_{\text {int }}$ is given by an asymptotic series of the form

$$
\mathcal{F}_{\text {int }}=\sum_{g \geq 2} \hbar^{2 g-2} \mathcal{F}^{(g)},
$$

the exponent $\mathcal{Z}_{\text {int }}=e^{\mathcal{F}_{\text {int }}}$ can be evaluated as the scalar product

$$
\mathcal{Z}_{\text {int }}=\langle\Sigma \mid \Omega\rangle \text {. }
$$

The states $\langle\Sigma|$ and $|\Omega\rangle$ belong to the completion of the Fock space associated with the $n_{B}$ branch points and defined by the relations

$$
\begin{aligned}
{\left[J_{p}^{[s]}, J_{q}^{\left[s^{\prime}\right]}\right] } & =p \delta_{p+q, 0} \delta_{s, s^{\prime}}, & & \left(s=1, \ldots, n_{B} ; p \in 2 \mathbb{Z}+1\right) \\
\left\langle 0_{\mathrm{tw}}\right| J_{-p}^{[s]} & =J_{p}^{[s]}\left|0_{\mathrm{tw}}\right\rangle=0 & & \left(s=1, \ldots, n_{B} ; p=1,3,5, \ldots\right) .
\end{aligned}
$$

The left state in (3.41),

$$
\langle\Sigma| \stackrel{\text { def }}{=}\left\langle 0_{\mathrm{tw}}\right| \exp \left(\frac{1}{2} \sum_{s, s^{\prime}=1}^{n_{B}} \sum_{p, q \geq 1} \frac{1}{p q} B_{p, q}^{\left[s, s^{\prime}\right]} J_{p}^{[s]} J_{q}^{\left[s^{\prime}\right]}+\frac{1}{\hbar} \sum_{s=1}^{n_{B}} \sum_{p \geq 5} \mu_{p}^{[s]} J_{p}^{[s]}\right),
$$

depends on the Bergmann kernel $B$ and the classical field $\Phi_{\mathrm{cl}}$ through

$$
\begin{aligned}
B_{p, q}^{\left[s, s^{\prime}\right]} & =\operatorname{Res}_{z \rightarrow a_{s}} \operatorname{Res}_{z^{\prime} \rightarrow a_{s^{\prime}}} \xi_{(z, s)}^{p} \xi_{\left(z^{\prime}, s^{\prime}\right)}^{q} B\left(z, z^{\prime}\right), & & p, q=1,3,5, \ldots ; \\
\mu_{p}^{(s)} & =\operatorname{Res}_{z \rightarrow a_{s}} y(z) d x(z) \xi_{(z, s)}^{-p}, & & p=5,7, \ldots
\end{aligned}
$$

The right state is in (3.41),

$$
|\Omega\rangle \stackrel{\text { def }}{=} \prod_{s=1}^{n_{B}} \exp \left(\sum_{n \geq 1} \frac{(-1)^{n}}{n !} \sum_{p_{1}, \ldots, p_{n}} \hbar^{2 g-2+n} \frac{w_{p_{1}, \ldots, p_{n}}^{(g)}}{p_{1} \ldots p_{n}} J_{-p_{1}}^{[s]} \ldots J_{-p_{n}}^{[s]}\right),
$$

represents a product of dressing operators, associated with the branch points. The coefficients $w_{p_{1}, \ldots, p_{n}}^{(g)}$ are universal numbers and are given by the genus- $g, n$-loop correlation functions in the Kontsevich model (see appendix A).

Correlation functions. The Fock space representation extends to the correlation functions of the collective field. They are given by

$$
\left.\left\langle d \Phi_{\mathrm{qu}}\left(z_{1}\right) \ldots d \Phi_{\mathrm{qu}}\left(z_{n}\right)\right\rangle=\left\langle d \Phi_{\mathrm{qu}}\left(z_{1}\right) \ldots d \Phi_{\mathrm{qu}}\left(z_{n}\right)\right\rangle_{\text {gauss }}+\left\langle d \Phi_{\mathrm{qu}}\left(z_{1}\right) \ldots d \Phi_{\mathrm{qu}}\left(z_{n}\right)\right\rangle_{\mathrm{i} h \mathrm{t}} 3.46\right)
$$

where \langle\rangle$_{\text {gauss }}$ is the result of all possible gaussian contractions with the Bergman kernel and

$$
\left\langle d \Phi_{\mathrm{qu}}\left(z_{1}\right) \ldots d \Phi_{\mathrm{qu}}\left(z_{n}\right)\right\rangle_{\mathrm{int}}=\sum_{s_{i}=1}^{n_{B}} \sum_{p_{i} \geq 1, \text { odd }} B_{p_{1}}^{\left[s_{1}\right]}\left(z_{1}\right) \ldots B_{p_{n}}^{\left[s_{n}\right]}\left(z_{n}\right)\left\langle\Sigma\left|J_{p_{1}}^{\left[s_{1}\right]} \ldots J_{p_{n}}^{\left[s_{n}\right]}\right| \Omega \nmid 3.47\right)
$$




\subsection{Diagram technique}

Performing the gaussian contractions we obtain an expression of the partition function as a series of Feynman graphs made of the following elements:

- Propagator:

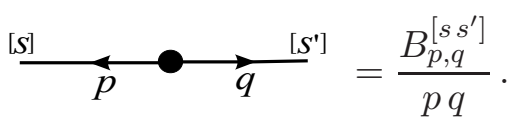

- Tadpole:

$$
\stackrel{[S]}{\rightleftarrows}=-\frac{1}{\hbar} \frac{\mu_{p}^{[s]}}{p}
$$

- Vertices:

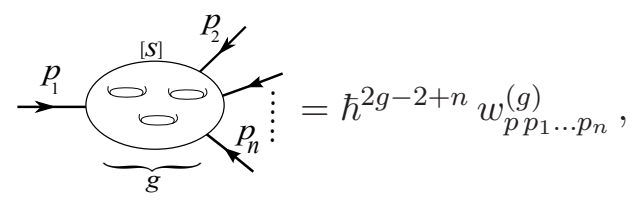

The tadpoles and the vertices are associated with a given branch point and carry label $s$, but their weights are given by universal numbers. The Feynman graphs are made out of these elements by connecting the open lines respecting the orientation. The orientation of the lines is such that vertices connect to tadpoles or propagators, but not directly with other vertices. The genus $g$ free energy is a sum of all connected Feynman graphs of genus $g$. The factors $(-1)^{n}$ in the dressing operator are taken into account by taking the tadpole with minus sign.

The diagram expansion for the correlation functions (3.46) is obtained by adding to the Feynman rules the propagators

$$
\begin{aligned}
& z \llbracket \quad z^{\prime}=B\left(z, z^{\prime}\right) \\
& \underset{p}{[S]} \bigcirc z=B_{p}^{[s]}(z)
\end{aligned}
$$

The Feynman rules (3.48)-(3.50) generalize those obtained in the one-matrix model [15] and for the scaling limit of various matrix models in [37-40]. The structure of the genus expansion is such that in the case of several branch points the partition function decomposes to a product of Kontsevich $\tau$-functions, related by gaussian correlations. Similar decomposition formulas for the case of the hermitian matrix model were suggested in [41-44].

It is possible, as is the case for the $O(n)$ matrix model [45], that the spectral curve has a symmetry relating different sheets of the Riemann surface. If such a symmetry is present, one should identify the twisted vacua associated with the branch points which belong to the same orbit of the symmetry. 


\section{Topological recursion}

\subsection{Summary}

Here we summarize the definitions and the rules of the method called topological recursion $[16-26]$.

Loop observables. Given the spectral curve (2.1), we define the $n$-point, genus $g$, functions $W_{n}^{(g)}\left(z_{1}, \ldots, z_{n}\right)$ as follows:

$$
\begin{aligned}
W_{1}^{(0)}(z) & \equiv y(z) d x(z), \\
W_{2}^{(0)}\left(z_{1}, z_{2}\right) & \equiv B\left(z_{1}, z_{2}\right)
\end{aligned}
$$

and recursively $W_{n+1}^{(g)}\left(z_{0}, z_{I}\right) \equiv W_{n+1}^{(g)}\left(z_{0}, z_{1}, \ldots, z_{n}\right)$ as

$$
W_{n+1}^{(g)}\left(z_{0}, z_{I}\right)=-\sum_{s=1}^{n_{B}} \operatorname{Res}_{z \rightarrow a_{s}} K\left(z_{0}, z\right)\left[\sum_{m, j} \sum_{J \subset I} W_{j+1}^{(m)}\left(z, z_{J}\right) W_{n-j+1}^{(g-m)}\left(\tilde{z}, z_{I \backslash J}\right)+W_{n+2}^{(g-1)}\left(z, \tilde{z}, z_{I}\right)\right] .
$$

The sum inside the brackets goes over $0 \leq m \leq g, 0 \leq j \leq n,(m, j) \neq(0,0),(g, n)$, and all possible ways to split the variables $z_{I}=\left\{z_{1}, \ldots, z_{n}\right\}$ into two sets, $z_{J}$ and $z_{I \backslash J}$. The r.h.s. is a sum of terms associated with the branch points $a_{s}$ of the Riemann surface $\Sigma$. Near each branch point $\tilde{z} \neq z$ is defined so that $x(\tilde{z})=x(z)$. The recursion kernel $K$ is defined in the vicinity of the branch point $z=a_{s}$ as

$$
K\left(z_{0}, z\right)=K\left(z_{0}, \tilde{z}\right) \stackrel{\text { def }}{=} \frac{\int_{\tilde{z}}^{z} B\left(z, z_{0}\right)}{2(y(z)-y(\tilde{z})) d x(z)}, \quad z \text { close to } a_{s} .
$$

The result of the integration does not depend on the choice of the local coordinate near $a_{s}$.

Free energy. The free energy $\mathcal{F}$ is defined as the formal (generically asymptotic) series

$$
\mathcal{F} \equiv \sum_{g=0}^{\infty} \hbar^{2-2 g} \mathcal{F}^{(g)}
$$

whose coefficients are expressed in terms of the loop observables and the elements of the spectral curve. $\mathcal{F}^{(0)}$ and $\mathcal{F}^{(1)}$ are respectively the classical action and the gaussian fluctuations of the bosonic field on the Riemann surface and for $g>1$ :

$$
\mathcal{F}^{(g)} \equiv \frac{1}{2-2 g} \sum_{s} \operatorname{Res}_{z \rightarrow a_{s}} S(z) W_{1}^{(g)}(z)
$$

where $d S(z)=y(z) d x(z)$.

Homogeneity. Under a rescaling of the one form $y d x$, the free energies turn into

$$
\mathcal{F}^{(g)}[\lambda y d x]=\lambda^{2-2 g} \mathcal{F}^{(g)}[y d x]
$$

for any $\lambda \in \mathbb{C}$. 
Symplectic invariance. For $g>1$, the free energy $\mathcal{F}^{(g)}$ is invariant under canonical transformations preserving the Poisson bracket (2.6).

\subsection{Topological recursion in the vicinity of a branch point}

The recursion equation (4.2) involves a sum over the branch points of the Riemann surface $\Sigma$. If we take the point $z_{0}$ close to one of the branch points and take an appropriate scaling limit, the recursion kernel will consist of a single term, associated with this branch point. The piece of the algebraic curve which survives in the scaling limit is a curve with only one simple branch point and genus zero. This is the complex curve for the Kontsevich model. We will see that the solution of the recursion equation for the Kontsevich model plays the role of a building block for the topological recursion on an arbitrary spectral curve.

\subsubsection{Solution of the recursion equation for the Kontsevich model}

The spectral curve for the Kontsevich integral is a genus 0 spectral curve with one simple branch point at $x=x_{0}$ and singular point at $x=\infty$. It can thus be described by a rational parameterization

$$
\mathcal{E}:\left\{\begin{array}{l}
x(z)=\frac{1}{2} z^{2}+x_{0} \\
y(z)=\mu_{3} z+\mu_{5} z^{3}+\mu_{7} z^{5}+\ldots
\end{array}\right.
$$

The moduli of the spectral curve are the moments $\mu_{3}, \mu_{5}, \ldots$ and the position $x_{0}$ of the branch point. ${ }^{10}$ When we deform the Riemann surface, both the moments and the position of $x_{0}$ are varied. There is only one branch point at $x=x_{0}$ and the variable $\xi_{(z, 0)} \stackrel{\text { def }}{=}$ $\sqrt{2 x-2 x_{0}}=z$ gives a global parametrization of the curve.

The initial data for the topological recursion are thus the one-form

$$
W_{1}^{(0)}(z)=y(z) d x(z)=\sum_{p \geq 3, \text { odd }} \mu_{p} z^{p-1} d z
$$

(the sum goes over $p$ odd) and the two-form

$$
W_{2}^{(0)}\left(z_{1}, z_{2}\right)=\frac{d z_{1} d z_{2}}{\left(z_{1}-z_{2}\right)^{2}}
$$

Let us write down the topological recursion in these terms. The recursion equation for the spectral curve (4.6) reads

$$
W_{k+1}^{(g)}\left(z_{0}, z_{K}\right)=-\operatorname{Res}_{z \rightarrow 0} K\left(z_{0}, z\right)\left[\sum_{m, j, J} W_{j+1}^{(m)}\left(z, z_{J}\right) W_{k-j+1}^{(g-m)}\left(\tilde{z}, z_{K / J}\right)+W_{k+2}^{(g-1)}\left(z, \tilde{z}, z_{K}\right)\right],
$$

where $\tilde{z}=-z$ and the recursion kernel (4.3) given by the series

$$
\begin{aligned}
K\left(z_{0}, z\right) & =\frac{d z_{0}}{4 y(z) d x(z)} \frac{2 z}{z^{2}-z_{0}^{2}} \\
& =-\frac{d z_{0}}{2 \mu_{3} z_{0}^{2} z d z} \frac{1}{1-z^{2} / z_{0}^{2}} \frac{1}{1+\sum_{p \geq 3, \text { odd }}\left(\mu_{p} / \mu_{3}\right) z^{p-3}}
\end{aligned}
$$

\footnotetext{
${ }^{10}$ Of course, the point $x_{0}$ can be placed at the origin by a global conformal transformation.
} 
Since the residue is taken when $z \rightarrow 0$ at fixed $z_{0}$, we have to expand in the positive powers in $z$. Apart of the overall power $1 / z$, the expansion is a Taylor series in $z^{2}$. If the r.h.s. of the recursion equation contains only finite number of negative powers of $z$, which we will see to be the case, then the residue picks only finite number of terms.

Let us demonstrate how the recursion equation works in the lower orders. We will evaluate $W_{3}^{(0)}$ and $W_{1}^{(1)}$. Expanding

$$
B\left(z, z_{1}\right)=\frac{d z d z_{1}}{z_{1}^{2}}\left(1+2 \frac{z}{z_{1}}+3 \frac{z^{2}}{z_{1}^{2}}+\ldots\right),
$$

we find

$$
\begin{aligned}
W_{3}^{(0)}\left(z_{0}, z_{1}, z_{3}\right) & =-\operatorname{Res}_{z \rightarrow 0} K\left(z_{0}, z\right)\left[B\left(z, z_{1}\right) B\left(-z, z_{2}\right)+B\left(-z, z_{1}\right) B\left(z, z_{2}\right)\right] \\
& =\frac{1}{\mu_{3}} \frac{d z_{0} d z_{1} d z_{2}}{z_{0}^{2} z_{1}^{2} z_{2}^{2}} \\
W_{1}^{(1)}\left(z_{0}\right) & =-\operatorname{Res}_{z \rightarrow 0} K\left(z_{0}, z\right) B(z,-z) \\
& =\frac{1}{8 \mu_{3} z_{0}^{4}}-\frac{\mu_{5}}{8 \mu_{3}^{2} z_{0}^{2}} .
\end{aligned}
$$

It is clear from the form of the kernel that the correlation functions with negative Euler characteristics, $2-2 g-n<0$, can be written under the form

$$
W_{n}^{(g)}\left(z_{1}, \ldots, z_{n}\right)=\mu_{3}^{2-2 g-n-l} \sum_{p_{i} \geq 1, \text { odd }} \prod_{i=1}^{n} \frac{d z_{i}}{z_{i}^{p_{i}+1}} \sum_{l \geq 0} \frac{1}{l !} \sum_{k_{j} \geq 3} \prod_{j=1}^{l}\left(-\frac{\mu_{k_{j}}}{k_{j}}\right) w_{k_{1}, \ldots, k_{l} \mid p_{1}, \ldots, p_{n}}^{(g)},
$$

where the summation goes over odd integer $p_{1}, \ldots, p_{n}$ and $k_{1}, \ldots, k_{l}$. The symmetry of the spectral curve $z \rightarrow z / \rho, \mu_{2 k} \rightarrow \rho^{2 k+1} \mu_{2 k}$ means that the observables (4.39) and the free energy do not change after this rescaling, which gives the restriction

$$
\sum_{i=1}^{l}\left(k_{j}-3\right)+\sum_{i=1}^{n}\left(p_{i}-3\right)=3(2 g-2)
$$

With these notations, the two examples considered above give:

$$
w_{1,1,1}^{(0)}=1 \quad, \quad w_{3 \mid 3}^{(1)}=\frac{3}{8} \quad, \quad w_{5 \mid 1}^{(1)}=\frac{5}{8} .
$$

\subsubsection{Open-close duality}

The form of the topological recursion obtained in the previous section involves both "open" moduli $z_{i}$, which we can visualize as boundaries on some world sheet, as well as the "closed" moduli $\mu_{p}(p \geq 3)$. The two types of moduli are related by the "loop insertion operator" [11], which depends non-linearly on the moments $\mu_{p}$ and the position of the branch point $x_{0}$. To make our derivation self-consistent, we prove here that the coefficients $\omega_{k_{J} \mid p_{I}}^{(g)}$ depend on the two groups of indices in a symmetric way:

$$
w_{k_{1}, \ldots, k_{l} \mid p_{1}, \ldots, p_{m}}^{(g)}=w_{k_{1}, \ldots, k_{k}, p_{1}, \ldots p_{m}}^{(g)} .
$$


By analogy with the string states we call this property open-close duality.

The open-close duality follows from the study of the variations of the correlation functions when the initial condition $y d x$ is perturbed. Consider the variation

$$
y d x(z) \rightarrow y d x(z)+\epsilon \frac{B\left(z, z_{*}\right)}{d z_{*}}
$$

for some $z_{*} \in \mathbb{C}$ and $\epsilon$ small enough. This can be translated into a shift in the moduli $\mu_{3}, \mu_{5}, \ldots$ :

$$
\mu_{p} \rightarrow \mu_{p}-\epsilon p z_{*}^{-p-1}, \quad p=3,5, \ldots
$$

Therefore the variation represents another background with the same position of the branch point and the same Bergman kernel, for which we can write the same loop equations.

On the other hand, using that by definition of $B=W_{2}^{(0)}$, it is easy to show [22] that the correlation functions transform as

$$
W_{n}^{(g)}\left(z_{1}, \ldots, z_{n}\right) \rightarrow W_{n}^{(g)}\left(z_{1}, \ldots, z_{n}\right)+\epsilon \frac{W_{n+1}^{(g)}\left(z_{*}, z_{1}, \ldots, z_{n}\right)}{d z_{*}}+O\left(\epsilon^{2}\right) .
$$

Comparing the two ways to write the linear term in $\epsilon$ in the r.h.s. of the recurrence equation, we prove (4.17). For example, instead of (4.16), we will write

$$
w_{1,1,1}^{(0)}=1 \quad, \quad w_{3,3}^{(1)}=\frac{3}{8} \quad, \quad w_{5,1}^{(1)}=\frac{5}{8} .
$$

\subsubsection{The topological recursion for the open coefficients: a trivalent theory}

Consider the simplest curve of the class (4.6), for which $\mu_{3} \neq 0$ ans $\mu_{5}=\mu_{7}=\cdots=0$. In this case the recursion kernel is

$$
K\left(z_{0}, z\right)=-\frac{1}{2 \mu_{3} z d z} \frac{1}{z_{0}^{2}-z^{2}} .
$$

Assume that the loop amplitudes have the expansion

$$
W_{n}^{(g)}\left(z_{1}, \ldots, z_{n}\right)=\mu_{3}^{2-2 g-n} \sum_{\left\{p_{i} \geq 1, \text { odd }\right\}} w_{p_{1}, \ldots, p_{n}}^{(g)} \prod_{i=1}^{n} \frac{d z_{i}}{z_{i}^{p_{i}+1}} .
$$

First we notice that if all functions are even in their arguments, then only the even part of the amplitude $W_{2}^{(0)}$ (the Bergman kernel) will contribute to the residue. This is so if one of the arguments of $W_{2}^{(0)}$ is an external variable. If one of the arguments, say $z_{1}$, is external, then we have to expand assuming $|z|<\left|z_{1}\right|$ and neglect the odd part of the expansion:

$$
W_{2}^{(0)}\left(z, z_{1}\right)=\frac{d z d z_{1}}{\left(z-z_{1}\right)^{2}} \rightarrow \frac{1}{z z_{1}} \sum_{p \geq 1}^{\infty} p z^{p} z_{1}^{-p}, \quad|z|<\left|z_{1}\right|
$$

where the sum goes over the odd $p$. Thus we can write for $W_{2}^{(0)}$ an expansion of the form (4.21), but containing both positive and negative odd "momenta" $p$ and define the Bergmann kernel in "momentum space" as

$$
w_{p, p_{1}}^{(0)}=|p| \delta_{p+p_{1}, 0} \quad\left(p, p_{1} \in \mathbb{Z}\right) .
$$


When $n=0$ and $g=1$, the first term of the recurrence equation (4.9) vanishes, while the second term is $W_{2}^{(0)}(z,-z)=B(z,-z)$ :

$$
W_{2}^{(0)}(z,-z)=\frac{1}{4 z^{2}} \text {. }
$$

By expanding the topological recursion relation (4.9) and extracting the coefficient of $\mu_{3}^{2-2 g-n}$, one gets a recursion relation for the coefficients $w_{p, p_{1}, \ldots, p_{n}}^{(g)}$ with $2 g+n>2$ :

$$
w_{p, p_{I}}^{(g)}=\frac{1}{2} \sum_{q+k=p-3}\left(\sum_{h=0}^{g} \sum_{J \subset I} w_{q, p_{J}}^{(h)} w_{k, p_{I \backslash J}}^{(g-h)}+w_{q, k, p_{I}}^{(g-1)}\right) .
$$

Here we used the abbreviation $p_{I}=\left\{p_{1}, \ldots, p_{n}\right\}$ for the set of indices $I=\{1, \ldots, n\}$ and the sum goes over all possible ways to split the set $I$ into two non-overlapping subsets $J$ and $I \backslash J$. The solution of equation (4.25) can be expressed as a sum of connected Feynman-like diagrams with trivalent vertices. The vertex whose three legs are labelled by momenta $p, q, k$ pointing inwards imposes the restriction $p+q+k=3$. All internal vertices and propagators have weight 1 except the external lines, which have weight $|p|$. It will be convenient to indicate only the absolute value of the momenta and indicate the signs by arrows on the propagators. Then, representing the amplitudes $w_{p_{1}, \ldots, p_{n}}^{(g)}$ as blobs with $g$ handles and $n$ legs,

$$
w_{p_{1}, \ldots, p_{n}}^{(g)}=\stackrel{p_{1}}{p_{g}^{0}}
$$

the recurrence equation takes the form

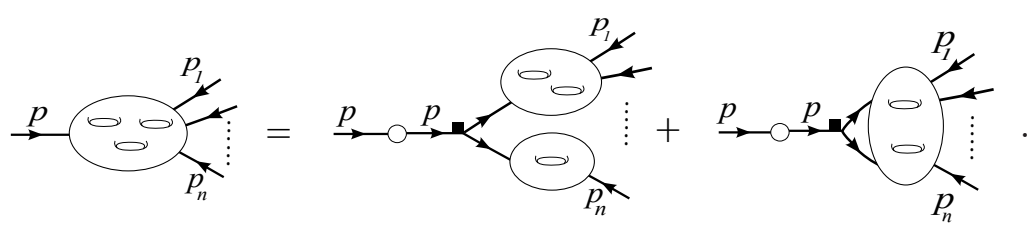

The vertex (with the given orientation of the lines) is

$$
\underset{k}{p}=\frac{\delta_{p+q+k+3,0}}{|p|} \text {. }
$$

The black square indicates the momentum whose absolute value is put in the denominator. The propagator is the genus zero two-point function (4.23)

$$
w_{p, q}^{(0)} \equiv \stackrel{p}{\rightarrow} \circ \longleftarrow^{q}=|p| \delta_{p+q, 0}=\stackrel{p}{\longrightarrow} \multimap \stackrel{p}{\longrightarrow} .
$$

The initial conditions are given by the genus zero two-point function (4.29) and the genus one tadpole, which is computed by (4.24):

$$
w_{p}^{(1)} \equiv \stackrel{p}{\longrightarrow}=\stackrel{p}{\rightarrow} \rightarrow \longrightarrow
$$




\section{Examples:}

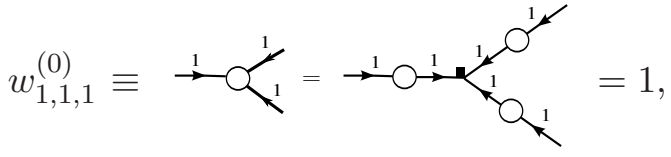

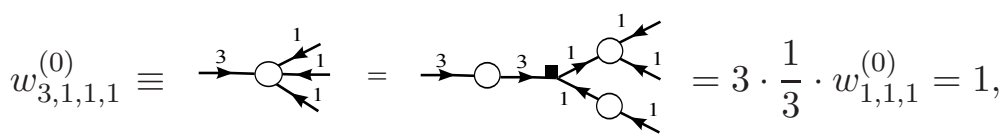

$$
\begin{aligned}
& w_{3,3}^{(0)} \equiv \overrightarrow{3} \bigcirc-\frac{3}{3}=\stackrel{3}{-\infty}=3 \cdot \frac{1}{3} \cdot w_{3}^{(1)} \cdot 3=\frac{3}{8} \text {, }
\end{aligned}
$$

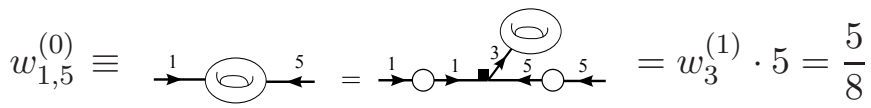

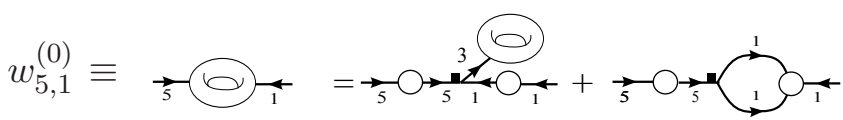

$$
\begin{aligned}
& =5 \cdot \frac{1}{5} \cdot\left(w_{3}^{(1)}+\frac{1}{2} \cdot w_{1,1,1}^{(0)}\right)=\frac{1}{8}+\frac{1}{2}=\frac{5}{8}
\end{aligned}
$$

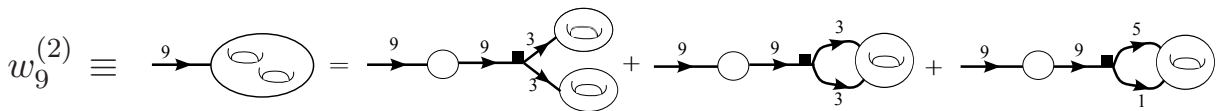

$$
\begin{aligned}
& =9 \cdot \frac{1}{9} \cdot\left(\frac{1}{2}\left(w_{3}^{(1)}\right)^{2}+\frac{1}{2} w_{3,3}^{(1)}+w_{5,1}^{(1)}\right)=\frac{1}{2}\left(\frac{1}{8^{2}}+\frac{3}{8}+2 \frac{5}{8}\right)=\frac{105}{128} .
\end{aligned}
$$

The recurrence equation resembles the mean field equation for a theory with cubic potential. This analogy can be made exact if one thinks of the string field theory Hamiltonian, which describes the elementary processes of splitting and joining of strings. Therefore the solution is not a series of Feynman diagrams, but have a tree-like structure, even though they have loops. This means that any diagram has a root (the starting point of the iteration) and the vertices are labeled by their height (the distance from the root of the tree). Because of this structure, the number of graph grows much slower that the number of Feynman graphs as the number of loops and external legs increases. These recurrence equations are of course equivalent to those obtained in topological gravity [46-48].

\subsubsection{Topological recursion for generic values of the moduli}

Once we know the coefficients $w_{p_{1}, \ldots, p_{n}}^{(g)}$, from the general form (4.14) of the solution and the open-closed duality it follows that we can write down the free energy and the correlators for any background, characterized by the moments $\mu_{5}, \mu_{7}, \ldots$ by allowing lines with tadpoles. Consider the generating function

$$
F^{(g)}\left[\left\{t_{p}\right\}\right]=\sum_{n \geq 1} \frac{1}{n !} \mu_{3}^{2-2 g-n} \sum_{p_{1}, \ldots, p_{n}} w_{p_{1}, \ldots, p_{n}}^{(g)} \frac{t_{p_{1}}}{p_{1}} \ldots \frac{t_{p_{n}}}{p_{n}} .
$$

The loop amplitudes (4.39) are obtained by applying the "loop insertion operator"

$$
\hat{w}(z)=\sum_{p \geq 1} \frac{1}{z^{p+1}} p \frac{\partial}{\partial t_{p}}
$$


to the free energy $F^{(g)}\left[\left\{t_{p}\right\}\right]$ :

$$
\begin{aligned}
W_{n}^{(g)}\left(z_{1}, \ldots, z_{n}\right) & =\left.d z_{1} \ldots d z_{n} \hat{w}\left(z_{1}\right) \ldots \hat{w}\left(z_{n}\right) F^{(g)}\left[\left\{t_{p}-\mu_{p}\right\}\right]\right|_{t_{p}=0} \\
& =\sum_{p_{i} \geq 1} W_{p_{1}, \ldots, p_{n}}^{(g)} \frac{d z_{1}}{z_{1}^{p_{1}+1}} \ldots \frac{d z_{n}}{z_{n}^{p_{n}+1}} .
\end{aligned}
$$

The new coefficients are graphically represented as follows:

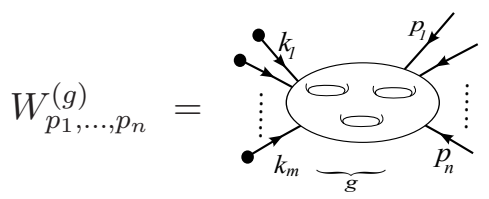

where the black blobs represent the moments $\mu_{p}$ :

$$
\stackrel{p}{\longleftrightarrow}=-\frac{\mu_{p}}{p} \quad(p=5,7, \ldots) .
$$

The above result can be obtained directly from the recursion equation with the general recursion kernel (4.10). By expanding the last factor in (4.10) we obtain the recurrence equation (4.27) with a modified vertex, which can be graphically represented as a sum of all possible insertions:

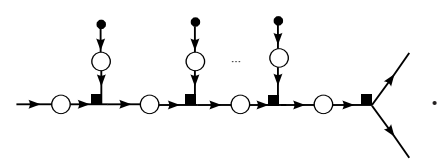

The same effect will be obtained if we consider each of the insertions in the dressed vertex (4.36) as the result of a recursion with the original vertex, with one of the amplitudes given by the tadpole

$$
\stackrel{p}{\leftrightarrow} \stackrel{p}{\hookrightarrow}=-\mu_{p}
$$

In particular the genus $g$ free energy for an arbitrary background $\left\{\mu_{p}\right\}$ is

$$
\mathcal{F}^{(g)}=\sum_{n \geq 1} \frac{1}{n !} \mu_{3}^{2-2 g-n} \sum_{p_{1}, \ldots, p_{n}} w_{p_{1}, \ldots, p_{n}}^{(g)} \frac{-\mu_{p_{1}}}{p_{1}} \ldots \frac{-\mu_{p_{n}}}{p_{n}} .
$$

\subsection{Topological recursion for an arbitrary spectral curve}

Now let us consider an arbitrary spectral curve with $n_{B}$ branch points $\left\{a_{s}\right\}_{i=1, \ldots, n_{B}}$ as defined in section 2 . We can cover the Riemann surface by an atlas of chartes associated with the local coordinates $\xi_{(z, s)}, s=1, \ldots, n_{B}$. The coordinate $\xi_{(z, s)}$ parametrizes the vicinity of the branch point $z=a_{s}$.

In order to write down the topological recursion (4.2) in a closed form, we need to expand the loop amplitudes in the local coordinates. Assume that the points $z_{1}, \ldots, z_{n}$ 
are close respectively to $a_{s_{1}}, \ldots, a_{s_{n}}$. Then, assuming that $2 g-2+n>0$, the $n$-point amplitude is expanded as a sum over odd integer momenta $p_{1}, \ldots, p_{n}$ :

$$
\left.W_{n}^{(g)}\left(z_{1}, \ldots, z_{n}\right)\right|_{z_{i} \rightarrow a_{s_{i}}}=\sum_{p_{i} \geq 1} W^{(g)}{ }_{p_{1} \ldots p_{n}}^{\left[s_{1} \ldots s_{n}\right]} \frac{d \xi_{\left(z_{1}, s_{1}\right)}}{\xi_{\left(z_{1}, s_{1}\right)}^{p_{1}+1}} \ldots \frac{d \xi_{\left(z_{n}, s_{n}\right)}}{\xi_{\left(z_{n}, s_{n}\right)}^{p_{n}+1}} .
$$

We will represent graphically the amplitudes $W_{p p_{1} \ldots p_{n}}^{(g)}$ as

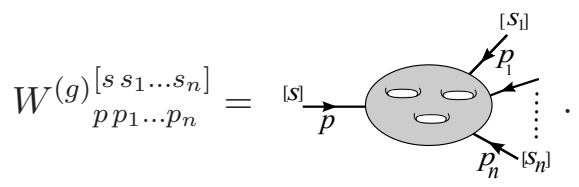

The momenta $p_{i}$ are associated with the oriented external legs and the labels $\left[s_{i}\right]$ are associated with the extremities of the legs.

Consider first a background with $\mu_{p}^{[s]}=\delta_{p, 3}$. Then the recurrence equation for the amplitudes is

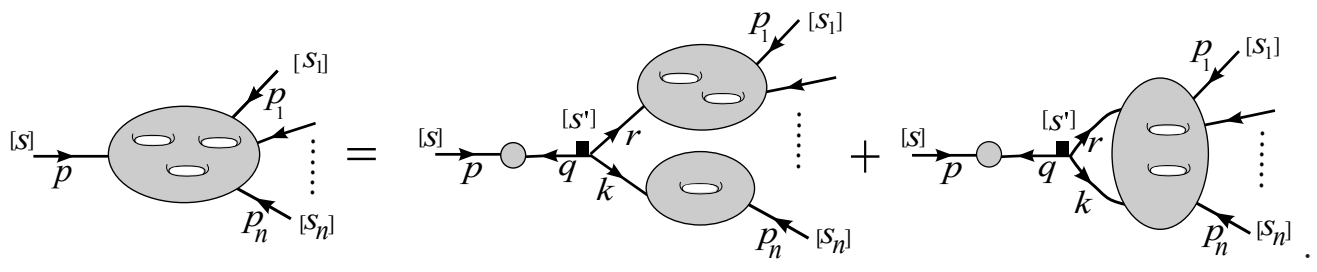

where in the right hand side one sums over $s^{\prime}=1, \ldots, n_{B}$ and all odd integers $q, k$ and $r$. The propagator and the vertex are defined as

$$
\begin{gathered}
{[s] \rightarrow \underset{q}{\leftarrow}\left[s^{\prime}\right] \equiv B_{-p,-q}^{\left[s, s^{\prime}\right]}=\operatorname{Res}_{z \rightarrow a_{s}} \operatorname{Res}_{z \rightarrow a_{s^{\prime}}} \frac{B\left(z, z^{\prime}\right)}{\xi_{(z, s)}^{p} \xi_{\left(z^{\prime}, s^{\prime}\right)}^{q}},} \\
\underbrace{p}_{k}=\frac{\delta_{p+q+k+3,0}}{|p|} .
\end{gathered}
$$

The propagator consists of two terms,

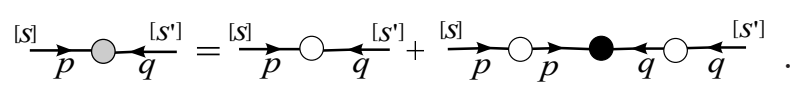

The first term is the diagonal part (4.29), which conserves the momentum:

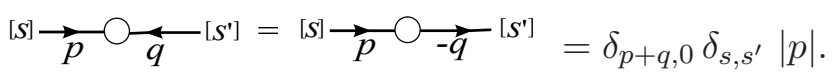

The second term is nonzero when $p$ and $q$ are positive, which is illustrated by changing the orientation of the second line,

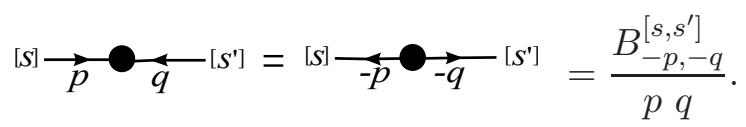

We used the same graphical notations as in the previous section; the white blobs are added to compensate the factors $1 / p$ and $1 / q$ in the definition of the black blob.

Finally, the genus one tadpole, which is computed by (4.24) does not depend on $s$ :

$$
W_{p}^{(1)} \equiv \stackrel{p}{\longrightarrow}=\stackrel{p}{\longrightarrow} \longrightarrow=\frac{1}{8} \delta_{p, 3} .
$$




\subsubsection{Loop insertion operator}

For a generic background $\left\{\mu_{i}^{[s]}\right\}$, the expansion of the correlation functions near a set of branch points $a_{s_{1}}, \ldots, a_{s_{n}}$ reads

$$
\left.W_{n}^{(g)}\left(z_{1}, \ldots, z_{n}\right)\right|_{z_{i} \rightarrow a_{s_{i}}}=\sum_{p_{i} \geq 1} \sum_{m \geq 0} \sum_{k_{j} \geq 3, \text { odd }} \frac{1}{m !} W_{p_{1} \ldots p_{n} \mid k_{1} \ldots k_{m}}^{(g)} \prod_{i=1}^{\left[s_{1} \ldots s_{n}\right]\left[s_{1}^{\prime} \ldots s_{m}^{\prime}\right]} \prod_{\xi_{\left(z_{i}, s_{i}\right)}^{n}} \frac{d \xi_{\left(z_{i}, s_{i}\right)}}{p_{j=1}^{p_{i}}} \prod^{m}\left(-\frac{\mu_{k_{j}}^{\left[s_{j}^{\prime}\right]}}{k_{j}}\right) .
$$

As in the preceding case, one can add one variable in the correlation functions by using the loop insertion operator consisting in the shift of the differential $y d x$ by the Bergman kernel:

$$
y d x(z) \rightarrow y d x(z)+\epsilon \frac{B\left(z, z_{*}\right)}{d z_{*}} .
$$

The only difference with the preceding section is that this operator acts as a shift on the times around all the branch points at once. Indeed, it acts as

$$
\mu_{k}^{[s]} \rightarrow \mu_{k}^{[s]}-\epsilon \frac{k}{\xi_{\left(z_{*}, s\right)}^{k+1}} \quad \text { for all } \quad s=1, \ldots, n_{B} .
$$

Applying the loop insertion operator to the genus $g$ free energy, with the insertion point close to a branch point $a_{s}$ gives the open closed duality

$$
W^{(g)}{ }_{p_{1} \ldots p_{n} \mid k k_{1} \ldots k_{m}}^{\left[s_{1} \ldots s_{n}\right]\left[s s_{1}^{\prime} \ldots s_{m}^{\prime}\right]}=W^{(g)} \underset{k p_{1} \ldots p_{n} \mid k_{1} \ldots k_{m}}{\left[s s_{1} \ldots s_{n}\right]\left[s_{m}^{\prime} \ldots s_{m}^{\prime}\right]} .
$$

\subsubsection{Topological recursion for generic background}

The open closed duality allows us to get the expression of the free energy in an arbitrary background characterized by the moments $\left\{\mu_{i}^{[s]}\right\}$ at the branch points $a_{s}, s=1, \ldots, n_{B}$, once we know the correlation functions of the trivial background $\mu_{p}^{[s]}=\delta_{p, 3}$. Indeed, the free energies

$$
\mathcal{F}^{(g)}=\sum_{n \geq 0} \frac{1}{n !} \sum_{p_{i} \geq 3, \text { odd }} \sum_{s_{i}=1}^{n_{B}} W_{p_{1} \ldots p_{n}}^{(g)} \prod_{i=1}^{\left[s_{1} \ldots s_{n}\right]}\left(-\frac{\mu_{p_{i}}^{\left[s_{i}\right]}}{p_{i}}\right) .
$$

are obtained by attaching tadpols

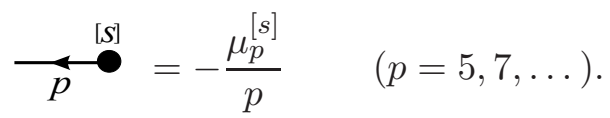

to the external legs.

\section{Relation between the two approaches}

Observables. The symplectic invariants defined by (4.1), (4.1) and (4.2) are related to the correlation functions of the CFT on the Riemann surface (3.47) by

$$
\left\langle d \Phi\left(z_{1}\right) \ldots d \Phi\left(z_{n}\right)\right\rangle_{\text {connected }}=\sum_{2 g+n>2} \hbar^{2 g-2+n} W^{(g)}\left(z_{1}, \ldots, z_{n}\right) .
$$

Furthermore, using (3.19), the recursion kernel can be written as

$$
K\left(z_{0}, z\right)=\frac{1}{2} \frac{\left\langle d \Phi_{\mathrm{qu}}\left(z_{0}\right) \Phi_{\mathrm{qu}}^{[s]}(z)\right\rangle_{\Sigma}}{d \Phi_{\mathrm{cl}}^{[s]}(z)}
$$


Recursion equation and conformal Ward identity. The recursion equation represents an integrated form of the Virasoro constraints. To see that, we write the recursion equation (4.9) as the operator identity

$$
2 d \Phi_{\mathrm{qu}}\left(z_{0}\right)+\sum_{s=1}^{n_{B}} \operatorname{Res}_{z \rightarrow a_{s}}\left(\left\langle d \Phi_{\mathrm{qu}}\left(z_{0}\right) \Phi_{\mathrm{qu}}^{[s]}(z)\right\rangle_{\Sigma} \frac{d \Phi_{\mathrm{qu}}(z) d \Phi_{\mathrm{qu}}(\tilde{z})}{d \Phi_{\mathrm{cl}}^{[s]}(z)}\right)=0 .
$$

Inserting this expression in the expectation value (5.1) one indeed obtains (4.9).

For $z_{0}$ close to the branch point $a_{s}$, the two-point function has Laurent expansion

$$
\left\langle d \Phi_{\mathrm{qu}}\left(z_{0}\right) \Phi_{\mathrm{qu}}^{[s]}(z)\right\rangle_{\Sigma}=d \xi_{\left(z_{0}, s\right)} \sum_{p \geq 1, \text { odd }} \xi_{\left(z_{0}, s\right)}^{-p-1} \xi_{(z, s)}^{p}+\text { regular. }
$$

Therefore the residue in (5.3) is a projection to the singular at $z_{0}=a_{s}$ part of the integrand. The recursion formula (5.3) implies that

$$
2 d \Phi_{\mathrm{qu}}(z)+\frac{d \Phi_{\mathrm{qu}}(z) d \Phi_{\mathrm{qu}}(\tilde{z})}{d \Phi_{\mathrm{cl}}^{[s]}(z)} \approx \frac{\left[d \Phi^{[s]}(z)\right]^{2}}{d \Phi_{\mathrm{cl}}^{[s]}(z)} \approx 0
$$

where $d \Phi^{[s]}=d \Phi_{\mathrm{cl}}^{[s]}+d \Phi_{\mathrm{qu}}^{[s]}$ and $\approx$ means 'equal up to a regular at $z=a_{s}$ function'. Since $d \Phi_{\mathrm{cl}}^{[s]}(z) \sim \xi_{(z, s)}^{2} d \xi_{(z, s)}$ when $z \rightarrow a_{s}$, (5.5) is equivalent to the conformal Ward identity (3.17).

Graph expansions. The trivalent graphs that appear in the recursion procedure and the Feynman graphs of the CFT approach must lead to the same result. Moreover, the Feynman rules of the CFT approach can be obtained by partial resummation of the trivalent graphs of the recursion procedure.

To see that, we first notice that, depending on the incoming and the outgoing momenta, only one of the two terms in the propagator (4.44) can be non-zero. Any trivalent graph can be split into connected subgraphs in such a way that the vertices belonging to the same subgraph can be connected only by Kontsevich (white) propagators, while the vertices belonging to two different subgraphs can be connected only by black propagators. Each such subgraph is associated with given branch point $a_{s}$ and contain only Kontsevich propagators (4.45). We can reorganize the sum over trivalent graphs by first summing up the contributions of the connected subgraphs associated with the branch points. We represent the sum of the contributions of all subgraphs with given values $p_{1}, \ldots, p_{n}$ of the external momenta by an effective vertex with $n$ legs and genus $g$ given by (3.36). For example,

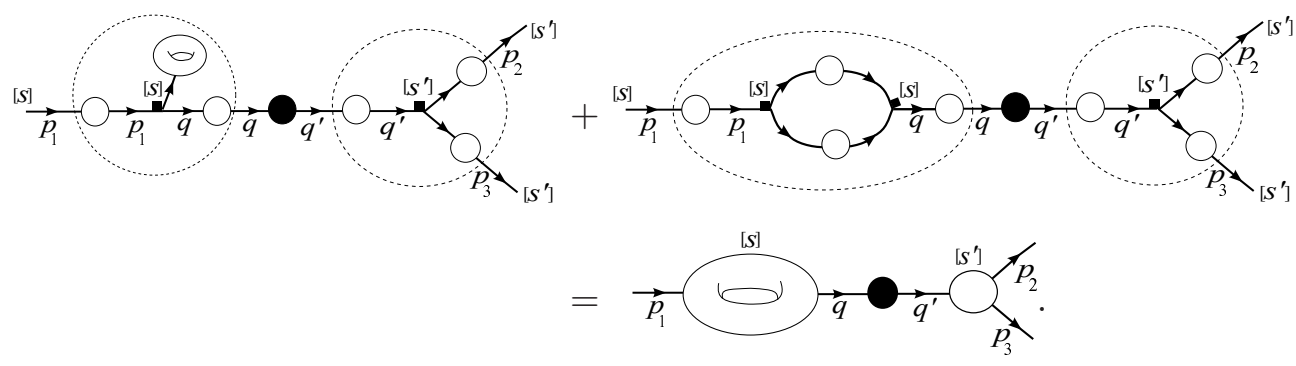


The partial resummation recasts the trivalent theory into an effective theory whose vertices are universal numbers, the amplitudes $w_{n_{1}, \ldots, n_{k}}^{(g)}$ of the Kontsevich model, and whose propagator and tadpole are given respectively by (3.48) and (3.49) with $\hbar=1$.

\section{Conclusion}

In this paper we reviewed and compared two approaches to quasiclassical expansion associated with a spectral curve: the CFT approach and the topological recursion (TR). The two approaches are formulated in their most general form. The only input is the spectral curve associated with the classical solution. Assuming that the collective field theory is conformal invariant, we derived a general formula for the genus expansion of the free energy and the current correlation functions.

We demonstrated that the diagram techniques obtained within the two approaches, although seemingly very different, lead to the same result for the genus expansion. More concretely, we showed that the CFT graph expansion is obtained as a partial resummation of the graph expansion in the TR.

The result of this paper proves that any theory solved by the topological recursion has a CFT dual living on the corresponding spectral curve. On the other hand, it provides a new way to prove that a system can be solved by the topological recursion. Namely, if a system, or matrix model, is solved by the CFT approach, this implies that it is solved by the topological recursion on the same spectral curve, which is can be more useful for explicit computations.

The quasiclassical expansion considered here can be applyed to systems with $\mathrm{U}(N)$ symmetry, where the eigenvalues of the matrix variables have fermionic statistics and the partition function is a $\tau$-function. In this case the quasiclassical expansion of the free energy contains only even powers of $\hbar=1 / N$. Our approach can be carried on also for non-trivial exchange statistics of the eigenvalues, which is the case for systems with $O(N)$ or $\operatorname{Sp}(N)$ symmetry and, more generally, for the $\beta$-ensembles with $\beta \neq 1$. In such systems the $1 / N$ expansion contains also odd powers in $1 / N$. The first orders were found in [49], whereas a TR formalism for hyperelliptical curves was derived in [50]. In order to generalize the CFT method to the case of $\beta$-ensembles, it is sufficient to add a term with a background charge $Q \sim \beta-1$ in the definition of the stress-energy tensor (3.16),

$$
T(z) d z^{2}=\frac{1}{2} \lim _{z^{\prime} \rightarrow z}\left[d \Phi(z) d \Phi\left(z^{\prime}\right)-B\left(z, z^{\prime}\right)-\frac{\beta-1}{\sqrt{2 \beta}} d^{2} \Phi\right] .
$$

This will change the right state $|\Omega\rangle$ in the operator representation (3.41), while the left state $\langle\Sigma|$ will remain the same.

Let us make some remarks concerning the application of the CFT/TR procedure to concrete matrix models. The case of the one-matrix model, where the spectral curve is hyperelliptic, is considered in details in [15]. In the case of the $O(n)$ model [45] and the ADE models [38] the spectral curve has a symmetry relating the branch points on different sheets of the Riemann surface. This makes it similar to a hyperelliptic curve. If such a 
symmetry is present, in the definition of the states (3.43) and (3.45), one should identify all branch points belonging to the same orbit.

In the case of the two-matrix model with potential $\operatorname{tr}\left[V_{1}(\mathbf{X})+\mathbf{X Y}+V_{2}(\mathbf{Y})\right]$, there is a symmetry $x \leftrightarrow y$ and one can construct a second Fock space representation of the partition function, based on the conformal invariance of the spectral plane of the matrix $\mathbf{Y}$. The two representations determine the same partition function. This non-trivial fact was proved on the TR side in $[19,51]$. The first Fock space representation is useful for computing the correlation functions of the matrix $\mathbf{X}$, while the second one is useful for computing the correlation functions of the matrix $\mathbf{Y}$. In this context a generalised topological recursion procedure was developed for computing the mixed trace correlation functions of the matrices $\mathbf{X}$ and $\mathbf{Y}$, or equivalently free energy in the case when the potential is an arbitrary polynomial of $\mathbf{X}$ and $\mathbf{Y}$ [52]. The CFT description of the $1 / N$ expansion for these correlation functions is still missing. For that one should construct a representation of the $W_{\infty}$ algebra on the complex curve. This is an interesting problem which we would like to address in the future.

\section{Acknowledgments}

Part of this work has been done during the visit of I.K. at the Departamento de Matemática, Instituto Superior Técnico, Lisboa. N.O. thanks B. Eynard for useful discussions and the Institut de Physique Théorique, CEA Saclay, for its kind hospitality .

\section{A Solving the Virasoro constraints near a branch point}

Here we obtain the solution to the Virasoro constraints (we have put $\hbar=1$ )

$$
L_{2 n}=\frac{1}{4} \sum_{k+q=2 n}:\left(J_{k}+\mu_{-k}\right)\left(J_{q}+\mu_{-q}\right):+\frac{1}{16} \delta_{n, 0},
$$

which determine the coefficients $w_{p_{1}, \ldots, p_{n}}^{(g)}$. We represent the oscillator amplitudes $J_{p}$ as

$$
\mu_{p}+J_{-p} \rightarrow t_{p}, \quad J_{p} \rightarrow p \frac{\partial}{\partial t_{p}} \quad(p \geq 1) .
$$

Then the Virasoro operators are represented by the differential operators

$$
\begin{aligned}
4 \hat{L}_{-2} & =\sum_{k \geq 1} k t_{k+2} \partial_{k} F+t_{1}^{2}, \\
4 \hat{L}_{0} & =2 \sum_{k \geq 1} k t_{k} \partial_{k} F+\frac{1}{4}, \\
4 \hat{L}_{p-3} & =\sum_{p-k-q=3} k q \partial_{k} \partial_{q}+2 \sum_{p+k-q=3} q t_{k} \partial_{q} \quad(p \geq 5, \text { odd })
\end{aligned}
$$

which act on the partition function $Z[t] \equiv Z\left[t_{1}, t_{3}, \ldots\right]$ :

$$
\hat{L}_{p-3} Z[t]=0, \quad p=1,3,5, \ldots
$$


We are interested in solution with $t_{3} \neq 0$. Then the $n=0$, or $p=3$, constraint is solved by assuming that the partion function is of the form

$$
Z\left[t_{p}\right]=t_{3}^{-1 / 24} e^{F\left[t_{p} / t_{3}^{p / 3}\right]}
$$

We look for a solution for the free energy $F$, which is a formal expansion in $t_{1}, t_{5}, t_{7}, \ldots$ and $1 / t_{3} \sim 1 / N$ :

$$
F=\sum_{g \geq 0} \sum_{n \geq 0} \frac{(-1)^{n}}{n !} \sum_{k_{1}, \ldots, k_{n} \geq 1} t_{3}^{2-2 g-n} w_{k_{1}, \ldots, k_{n}}^{(g)} \frac{t_{k_{1}} \ldots t_{k_{n}}}{k_{1} \ldots k_{n}} .
$$

The compatibility of this expansion with (A.5) implies that the coefficients $w_{k_{1}, \ldots, k_{n}}^{(g)}$ are nonzero only if

$$
\sum_{i=1}^{n} k_{i}=3(2 g-2+n)
$$

Written for the free energy $F=\ln Z$, , the Virasoro constraints become a set of quadratic constraints for the derivatives

$$
w_{p} \stackrel{\text { def }}{=}-p \partial_{p} F, \quad w_{p, q} \stackrel{\text { def }}{=} p q \partial_{p} \partial_{q} F .
$$

We have to solve for $p=1,3, \ldots$

$$
\sum_{p-k-q=3}\left(w_{k} w_{q}+w_{k, q}\right)-2 \sum_{p+k-q=3} t_{k} w_{q}+t_{1}^{2} \delta_{p, 1}=0 .
$$

In particular, the equation for $p=3$ means that the generating function

$$
\phi(z)=-\sum_{p \geq 1} \frac{w_{p}}{p z^{p}}+\sum_{p \geq 1} t_{p} \frac{z^{p}}{p}
$$

is scale invariant with respect to $t_{p} \rightarrow \rho^{-p} t_{p}, z \rightarrow \rho z$.

One can solve (A.9) recursively in the genus $g$ by expanding

$$
w_{p}\left[t_{k}\right]=\sum_{g \geq 1} t_{3}^{2-2 g} w_{p}^{(g)}\left[t_{k} / t_{3}\right] .
$$

The solution of (A.9) can be obtained as a sum of graphically represented by introducing a trivalent vertex which gives the conservation law of the three incoming momenta $p, q, k$ :

$$
v_{p, q, k}=\frac{1}{t_{3}} \delta_{p+q+k-3,0} .
$$

This vertex satisfies the selection rule (A.7) with $g=0$ and $n=3$. Then (A.9) takes the form

$$
w_{p}^{(g)}=v_{p,-k,-q}\left(\frac{1}{2} \sum_{h=0}^{g} w_{k}^{(h)} w_{q}^{(g-h)}+w_{k, q}^{(g-1)}\right)+2 v_{p, k,-q} t_{k} w_{q}^{(g)}+\frac{1}{2} v_{p, 1,1} t_{1}^{2} .
$$


This is the generating function for a set of recurrence equations for the coefficients $w_{p_{1}, \ldots, p_{n}}^{(g)}$. The equation for the correlator with $n+1$ legs $p, p_{1}, \ldots, p_{n}$ is obtained by differentiating w.r.t. $t_{p_{1}}, \ldots, t_{p_{n}}$ and then taking $t_{q}=\delta_{q, 3}$. The equation has the following graphical representation:

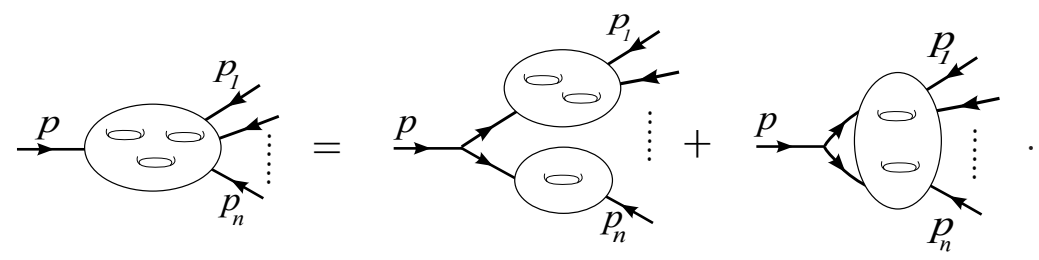

This graphical representation is related to that for the topological recursion (A.14) by replacing

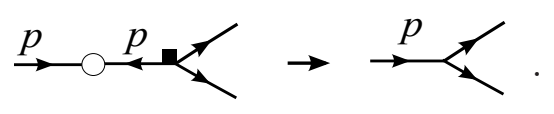

Open Access. This article is distributed under the terms of the Creative Commons Attribution Noncommercial License which permits any noncommercial use, distribution, and reproduction in any medium, provided the original author(s) and source are credited.

\section{References}

[1] R. Dijkgraaf and C. Vafa, Matrix models, topological strings and supersymmetric gauge theories, Nucl. Phys. B 644 (2002) 3 [hep-th/0206255] [SPIRES].

[2] V. Bouchard, A. Klemm, M. Mariño and S. Pasquetti, Remodeling the B-model, Commun. Math. Phys. 287 (2009) 117 [arXiv:0709.1453] [SPIRES].

[3] R. Dijkgraaf and C. Vafa, Toda theories, matrix models, topological strings and $N=2$ gauge systems, arXiv:0909.2453 [SPIRES].

[4] I.K. Kostov, The ADE face models on a fluctuating planar lattice, Nucl. Phys. B 326 (1989) 583 [SPIRES].

[5] I.K. Kostov, Solvable statistical models on a random lattice, Nucl. Phys. Proc. Suppl. 45A (1996) 13 [hep-th/9509124] [SPIRES].

[6] A. Marshakov, A. Mironov and A. Morozov, Generalized matrix models as conformal field theories: discrete case, Phys. Lett. B 265 (1991) 99 [SPIRES].

[7] S. Kharchev, A. Marshakov, A. Mironov, A. Morozov and S. Pakuliak, Conformal matrix models as an alternative to conventional multimatrix models, Nucl. Phys. B 404 (1993) 717 [hep-th/9208044] [SPIRES].

[8] I.K. Kostov, Conformal field theory techniques in random matrix models, hep-th/9907060 [SPIRES].

[9] I. Kostov, Matrix models a conformal field theories, in Les Houches 2004 "Applications of random matrices in physics", Springer, U.S.A. (2004).

[10] A.B. Zamolodchikov, Conformal scalar field on the hyperelliptic curve and critical Ashkin-Teller multipoint correlation functions, Nucl. Phys. B 285 (1987) 481 [SPIRES].

[11] J. Ambjørn, L. Chekhov, C.F. Kristjansen and Y. Makeenko, Matrix model calculations beyond the spherical limit, Nucl. Phys. B 404 (1993) 127 [hep-th/9302014] [SPIRES]. 
[12] R. Dijkgraaf, H.L. Verlinde and E.P. Verlinde, Loop equations and Virasoro constraints in nonperturbative 2D quantum gravity, Nucl. Phys. B 348 (1991) 435 [SPIRES].

[13] M. Fukuma, H. Kawai and R. Nakayama, Continuum Schwinger-Dyson equations and universal structures in two-dimensional quantum gravity, Int. J. Mod. Phys. A 6 (1991) 1385 [SPIRES].

[14] E. Verlinde and H. Verlinde, A solution of two-dimensional topological quantum gravity, Nucl. Phys. B 348 (1991) 457.

[15] I. Kostov, Matrix models as conformal field theories: genus expansion, Nucl. Phys. B 837 (2010) 221 [arXiv:0912.2137] [SPIRES].

[16] B. Eynard, Topological expansion for the 1-hermitian matrix model correlation functions, JHEP 11 (2004) 031 [hep-th/0407261] [SPIRES].

[17] B. Eynard and N. Orantin, Topological expansion of the 2-matrix model correlation functions: diagrammatic rules for a residue formula, JHEP 12 (2005) 034 [math-ph/0504058] [SPIRES].

[18] L. Chekhov and B. Eynard, Hermitean matrix model free energy: Feynman graph technique for all genera, JHEP 03 (2006) 014 [hep-th/0504116] [SPIRES].

[19] L. Chekhov, B. Eynard and N. Orantin, Free energy topological expansion for the 2-matrix model, JHEP 12 (2006) 053 [math-ph/0603003] [SPIRES].

[20] L. Chekhov and B. Eynard, Matrix eigenvalue model: Feynman graph technique for all genera, JHEP 12 (2006) 026 [math-ph/0604014] [SPIRES].

[21] B. Eynard, Formal matrix integrals and combinatorics of maps, math-ph/0611087 [SPIRES].

[22] B. Eynard and N. Orantin, Invariants of algebraic curves and topological expansion, math-ph/0702045 [SPIRES].

[23] B. Eynard and A.P. Ferrer, Topological expansion of the chain of matrices, JHEP 07 (2009) 096 [SPIRES].

[24] G. Borot and B. Eynard, Enumeration of maps with self avoiding loops and the $O(n)$ model on random lattices of all topologies, arXiv:0910.5896.

[25] B. Eynard and M. Mariño, A holomorphic and background independent partition function for matrix models and topological strings, arXiv:0810.4273 [SPIRES].

[26] B. Eynard and N. Orantin, Topological recursion in enumerative geometry and random matrices, J. Phys. A 42 (2009) 293001.

[27] I.M. Krichever, The tau function of the universal Whitham hierarchy, matrix models and topological field theories, Commun. Pure Appl. Math. 47 (1994) 437 [hep-th/9205110] [SPIRES].

[28] K. Takasaki and T. Takebe, Integrable hierarchies and dispersionless limit, Rev. Math. Phys. 7 (1995) 743 [hep-th/9405096] [SPIRES].

[29] K. Takasaki and T. Takebe, $\hbar$-expansion of KP hierarchy: recursive construction of solutions, arXiv:0912. 4867 [SPIRES].

[30] M. Bertola, Free energy of the two-matrix model/dToda $\tau$-function, Nucl. Phys. B 669 (2003) 435 [hep-th/0306184] [SPIRES].

[31] E. Verlinde and H. Verlinde, Chiral bosonization, determinants and the string partition function, Nucl. Phys. B 288 (1987) 357.

[32] V.G. Knizhnik, Multiloop amplitudes in the theory of quantum strings and complex geometry, Sov. Phys. Usp. 32 (1989) 945 [Usp. Fiz. Nauk 159 (1989) 401] [SPIRES]. 
[33] C. Vafa, Operator formulation on Riemann surfaces, Phys. Lett. B 190 (1987) 47 [SPIRES].

[34] Y. Matsuo, Moduli space, conformal algebra and operator formulation on a Riemann surface, Mod. Phys. Lett. A 3 (1988) 1 [SPIRES].

[35] R. Dijkgraaf, E.P. Verlinde and H.L. Verlinde, $C=1$ conformal field theories on Riemann surfaces, Commun. Math. Phys. 115 (1988) 649 [SPIRES].

[36] M. Kontsevich, Intersection theory on the moduli space of curves and the matrix Airy function, Commun. Math. Phys. 147 (1992) 1 [SPIRES].

[37] I.K. Kostov, Strings with discrete target space, Nucl. Phys. B 376 (1992) 539 [hep-th/9112059] [SPIRES].

[38] I.K. Kostov, Gauge invariant matrix model for the A-D-E closed strings, Phys. Lett. B 297 (1992) 74 [hep-th/9208053] [SPIRES].

[39] S. Higuchi and I.K. Kostov, Feynman rules for string field theories with discrete target space, Phys. Lett. B 357 (1995) 62 [hep-th/9506022] [SPIRES].

[40] I.K. Kostov and V.B. Petkova, Non-rational $2 D$ quantum gravity. II: target space CFT, Nucl. Phys. B 769 (2007) 175 [hep-th/0609020] [SPIRES].

[41] A. Givental, Semisimple Frobenius structures at higher genus, math/0008067v4.

[42] A. Alexandrov, A. Mironov and A. Morozov, BGWM as second constituent of complex matrix model, JHEP 12 (2009) 053 [arXiv:0906.3305] [SPIRES].

[43] A.S. Alexandrov, A. Mironov, A. Morozov and P. Putrov, Partition functions of matrix models as the first special functions of string theory. II. Kontsevich model, Int. J. Mod. Phys. A 24 (2009) 4939 [arXiv:0811.2825] [SPIRES].

[44] A.S. Alexandrov, A. Mironov and A. Morozov, Instantons and merons in matrix models, Physica D 235 (2007) 126 [hep-th/0608228] [SPIRES].

[45] I.K. Kostov, $O(n)$ vector model on a planar random lattice: spectrum of anomalous dimensions, Mod. Phys. Lett. A 4 (1989) 217 [SPIRES].

[46] R. Dijkgraaf, H.L. Verlinde and E.P. Verlinde, Loop equations and Virasoro constraints in nonperturbative 2D quantum gravity, Nucl. Phys. B 348 (1991) 435 [SPIRES].

[47] R. Dijkgraaf and H. L. Verlinde, Topological strings and loop equations, in Cargese 1990, Proceedings, Random surfaces and quantum gravity, Plenum Press, U.S.A. (1991).

[48] R. Dijkgraaf and E. Witten, Mean field theory, topological field theory, and multimatrix models, Nucl. Phys. B 342 (1990) 486 [SPIRES].

[49] P. Wiegmann and A. Zabrodin, Large- $N$ expansion of the $2 D$ Dyson gas, J. Phys. A 39 (2006) 8933 [hep-th/0601009] [SPIRES].

[50] L. Chekhov, B. Eynard and O. Marchal, Topological expansion of the Bethe ansatz and quantum algebraic geometry, arXiv:0911.1664 [SPIRES].

[51] B. Eynard and N. Orantin, Topological expansion of mixed correlations in the hermitian 2 matrix model and $x-y$ symmetry of the $F_{g}$ invariants, arXiv: 0705.0958 [SPIRES].

[52] B. Eynard and N. Orantin, Topological expansion and boundary conditions, JHEP 06 (2008) 037 [arXiv:0710.0223] [SPIRES]. 